\title{
Enhanced optical nonlinearities under collective strong light-matter coupling
}

\author{
Raphael F. Ribeiro, ${ }^{1}$ Jorge A. Campos-Gonzales-Angulo, ${ }^{1}$ Noel C. Giebink, ${ }^{2}$ Wei Xiong, ${ }^{1,3}$ and Joel Yuen-Zhou ${ }^{1}$ \\ ${ }^{1}$ Department of Chemistry and Biochemistry, University of California San Diego, La Jolla, CA 92093 \\ ${ }^{2}$ Department of Electrical Engineering, The Pennsylvania State University, University Park, PA, 16802 \\ ${ }^{3}$ Materials Science and Engineering Program, University of California San Diego, La Jolla, CA 92093
}

(Dated: June 16, 2020)

\begin{abstract}
Optical microcavities and metallic nanostructures have been shown to significantly modulate the dynamics and spectroscopic response of molecular systems. We present a study of the nonlinear optics of a model consisting of $N$ anharmonic multilevel systems (e.g., Morse oscillators) undergoing collective strong coupling with a resonant infrared microcavity. We find that, under experimentally accessible conditions, molecular systems in microcavities may have nonlinear phenomena significantly intensified due to the high quality of polariton resonances and the enhanced microcavity electromagnetic energy density relative to free space. Particularly large enhancement of multiphoton absorption happens when multipolariton states are resonant with bare molecule multiphoton transitions. In particular, our model predicts two-photon absorption cross section enhancements by several orders of magnitude relative to free space when the Rabi splitting $\Omega_{R}$ is approximately equal to the molecular anharmonic shift $2 \Delta$. Our results provide rough upper bounds to resonant nonlinear response enhancement factors as relaxation to dark states is treated phenomenologically. Notably, ensembles of two-level systems undergoing strong coupling with a cavity (described by the Tavis-Cummings model) show no such optical nonlinearity enhancements, highlighting the rich phenomenology afforded by multilevel anharmonic systems. Similar conclusions are expected to hold for excitonic systems that share features with our model (e.g., molecular dyes with accessible $S_{0} \rightarrow S_{1} \rightarrow S_{2}$ transitions) and strongly interact with a UV-visible cavity.
\end{abstract}

\section{INTRODUCTION}

Light-induced nonequilibrium phenomena is a topic of great contemporary interest due to its relevance to the energy, biochemical, and material sciences. Nonlinear spectroscopy provides tools for probing and controlling nonequilibrium quantum dynamics [1, 2] driven by external radiation. Applications of nonlinear optics to chemistry include investigations of the dynamics of energy and charge transport in light-harvesting complexes $[3,4]$, organic electronics [5], and other excitonic systems [6]. Nonlinear optical processes are also basic to various developing technologies including all-optical devices $[7,8]$, quantum information processors [9, 10], and enhanced sensors [11].

Unfortunately, the nonlinearities of molecular systems are generally weak [12]. Recently, hybrid materials consisting of a molecular ensemble hosted by a photonic (or plasmonic) device (e.g., optical microcavities and metallic nanostructures) have been explored as potential sources of magnified nonlinear optical response [13-16]. Under accessible experimental conditions (room temperature and atmospheric pressure) the light-matter interaction in photonic materials can become strong enough that excited states corresponding to superposition of (collective) material polarization and cavity excitations emerge [16-20]. The corresponding hybrid quasiparticles (modes) are commonly denoted by (cavity)-polaritons [21]. They show controllable coherence and relaxation dynamics that allow modulation of various physicochemical properties. Molecular phenomena significantly influenced by strong light-matter interactions include: energy transfer[22-24], charge and exci- ton transport [25-27], and chemical kinetics [28-31].
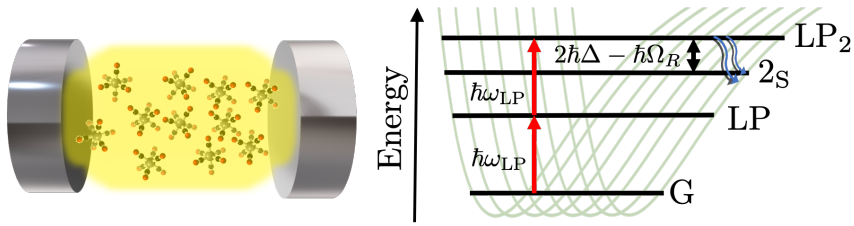

FIG. 1. Left: Planar microcavity consisting of two highly reflective mirrors filled with a molecular ensemble (e.g., $\mathrm{W}(\mathrm{CO})_{6}$ in solution) with sufficiently large collective oscillator strength that hybrid polaritonic states are formed. Right: Mechanism for enhancement of two-photon absorption by an ensemble of Morse oscillators (represented by the various illustrative Morse potentials) under strong coupling with an optical cavity. An external field resonant with the lowerpolariton (LP) drives the hybrid cavity and excites two-LP states which can be tuned to be near-resonant with the anharmonically shifted doubly-excited molecular states forming the totally-symmetric $2_{S}$ state. This polariton-mediated absorption channel allows enhancement of several orders of magnitude of the molecular two-photon absorption cross-section.

Recent experiments [32-41] have surveyed the nonlinear optics of polaritonic systems to gain further insight into the relaxation kinetics and optical response of strongly coupled devices. In Refs. [36-38, 42], the transient response and relaxation to equilibrium of vibrational polaritons (those arising from the strong coupling of molecular infrared polarization with a resonant microcavity) were investigated with pump-probe and twodimensional infrared spectroscopy. These studies demonstrated how vibrational anharmonicity is manifested in 
the pump-probe polariton response [43]. However, the observed time-resolved spectra were sensitive to various system-dependent effects arising from the small Rabi splittings of the studied materials, and significant static and dynamical disorder which induces ultrafast polariton decay into the weakly-coupled (dark) molecular modes.

In this work, we focus on universal (systemindependent) features of molecular polariton nonlinear optics. Our aim is to provide qualitative and quantitative insight on the potential to achieve giant optical nonlinearities with molecular polaritons in the collective regime (which is the case in most experiments) with a large number of molecules in a microcavity (nonlinear optical effects of single-molecule polaritonic systems have been studied within a non-adiabatic model of the dynamical Casimir effect in Ref. [44], as well as in vibrational polariton spectra in Ref. [45]).

In Sec. 2, we describe our model, provide an analytical expression for the nonlinear optical susceptibility of an ideal molecular ensemble under strong interaction with a microcavity (the full derivation is in the SI Sec. 2), and discuss its main features. In Sec. 3, we compare the free space and the polariton-mediated two-photon absorption (TPA) rates, and show that, especially when overtone polariton transitions are resonant with multiphoton molecular transitions, nonlinearity enhancements of several orders of magnitude may be achieved with currently available optical cavities (Fig. 1) as a result of three main effects: increased electromagnetic energy density in the optical microcavity relative to free space $[13,46]$, creation of new optical resonances, and strong coupling induced suppression of lineshape broadening [47]. A discussion of our main results and conclusions are given in Sec. 4. Our article is accompanied by Supporting Information (SI) containing detailed derivations of the molecular nonlinear susceptibility, and rate of nonlinear absorption in free space and under strong coupling with an optical microcavity.

\section{MOLECULAR NONLINEAR RESPONSE}

\subsection{Effective Hamiltonian}

The physical system of interest consists of a molecular ensemble containing $N$ molecules uniformly distributed in a region enclosed by two highly-reflective planar mirrors separated by a distance $L_{c}$ of the order of the wavelength of a specific material infrared excitation $\left(L_{c}\right.$ is usually between 0.1 and $20 \mu \mathrm{m}$ ) [48-50]. This setup corresponds to a Fabry-Perot (FP) microcavity [13, 46] filled with a homogeneous molecular system. Our description of the molecular subsystem will include explicitly only the modes which are nearly-resonant with the optical cavity. The effects of all other molecular degrees of freedom will be treated phenomenologically by introduction of damping to the molecular polarization (see below).

We suppose that the interaction between the cav- ity field and the molecular polarization $\sum_{i=1}^{N}\left\langle 1_{i}\left|p_{i}\right| 0_{i}\right\rangle$ (where $p_{i}$ is the effective dipole operator of the $i$ th molecule and $0_{i}$ and $1_{i}$ denotes states where the $i$ th molecule is in the ground and first excited-state, respectively, whereas all other molecules are in the groundstate) is significantly stronger than the coupling of either subsystem to external (bath) degrees of freedom, but still only a tenth or less of the bare vibrational and cavity frequencies (so considerations exclusive to ultrastrong coupling can be ignored [51-53]).

The total Hamiltonian of the composite material is given by $H_{T}(t)=H_{\mathrm{L}}(t)+H_{\mathrm{M}}+H_{\mathrm{LM}}$, where $H_{\mathrm{L}}(t)$ and $H_{\mathrm{M}}$ are the bare cavity (driven by an external timedependent field) and molecular Hamiltonians and $H_{\mathrm{LM}}$ contains the interaction between the cavity EM field and matter. The cavity Hamiltonian is given by:

$$
\begin{aligned}
H_{\mathrm{L}}(t)= & \sum_{\mathbf{k}} \hbar \omega_{\mathbf{k}} b_{\mathbf{k}}^{\dagger} b_{\mathbf{k}} \\
& +i \hbar \sqrt{\frac{\kappa}{2}} \sum_{\mathbf{k}}\left\{\left[b_{\mathbf{k i n}}^{\mathrm{L}}(t)\right]^{\dagger} b_{\mathbf{k}}-b_{\mathbf{k}}^{\dagger} b_{\mathbf{k i n}}^{\mathrm{L}}(t)\right\},
\end{aligned}
$$

where this effective Hamiltonian can be obtained from input-output theory [54-56] which describes the interaction of the optical cavity with left input and right output flux operators $b_{\text {kin }}^{\mathrm{L}}(t)$ and $b_{\text {kout }}^{\mathrm{R}}(t)$, respectively (SI Sec. I), and we include only a single cavity band and EM field polarization (as the cavity band gaps are much larger than the cavity and molecular linewidths, due to the smallness of the cavity's longitudinal length $L_{c}$, and electric field polarization conversion gives a tiny perturbation on the results presented here especially as we consider isotropic molecular ensembles [57]). The frequency of the mode with (in-plane) wave-vector $\mathbf{k}=\left(k_{x}, k_{y}\right)$ is $\omega_{\mathbf{k}}=c \sqrt{\mathbf{k}^{2}+m^{2} \pi^{2} / L_{c}^{2}} / n(m \in \mathbb{Z}$ is the index of the cavity band; $n$ is the index of refraction of the cavity interior; hereafter $n=1$ ), and $b_{\mathbf{k}}$ is its annihilation operator. The cavity leakage (decay) rate is $\kappa$. The Heisenberg equations of motion generated by Eq. 1 are turned into the Heisenberg-Langevin equations when the replacement $\omega_{\mathbf{k}} \rightarrow \tilde{\omega}_{\mathbf{k}} \equiv \omega_{\mathbf{k}}-i \kappa / 2$ is performed (SI Sec. $1)$.

The bare vibrational dynamics is generated by the Hamiltonian $H_{M}$ given by

$$
H_{\mathrm{M}}=\sum_{i=1}^{N} \hbar \omega_{0} a_{i}^{\dagger} a_{i}-\hbar \Delta \sum_{i=1}^{N} a_{i}^{\dagger} a_{i}^{\dagger} a_{i} a_{i},
$$

where the vibrational creation and annihilation operators of the $i$ th molecule are $a_{i}^{\dagger}$ and $a_{i}$, respectively. The fundamental frequency of each molecule is $\omega_{0}$, and the anharmonic coupling is $\Delta>0$. We neglect intermolecular interactions as they are too weak relative to lightmatter coupling (the situation could be different in other situations, e.g., molecular crystals and liquid-solid interfaces $[21,58])$. We treat the relaxation of the molecular subsystem phenomenologically by converting the Heisenberg equations of motion (EOMs) of molecular operators into Heisenberg-Langevin EOMs via the substitution 
$\omega_{0} \rightarrow \tilde{\omega}_{0}=\omega_{0}-i \gamma_{m} / 2$, where $\gamma_{m}$ is the bare molecule fundamental transition (homogeneous) linewidth.

The light-matter interaction is treated with the multipolar gauge [59] in the long-wavelength limit within the rotating wave approximation[55] (see next paragraph for a discussion of these and other approximations):

$$
H_{\mathrm{LM}}=-\sum_{\mathbf{k}} \sum_{i=1}^{N}\left(g_{i \mathbf{k}} a_{i}^{\dagger} b_{\mathbf{k}}+\bar{g}_{i \mathbf{k}} a_{i} b_{\mathbf{k}}^{\dagger}\right)+H_{\mathrm{P}^{2}},
$$

where $g_{j \mathbf{k}}=\mu_{j} \cdot E_{j \mathbf{k}}^{c}$ is the coupling constant for the interaction between the $j$ th molecular vibration (with effective transition dipole moment $\mu_{j}$ ) and the cavity mode $\mathbf{k}$, with mode profile evaluated at the position $\mathbf{r}_{j}$ of the $j$ th molecule, i.e., $E_{j \mathbf{k}}^{c}=i \sqrt{\hbar \omega_{\mathbf{k}} /\left(2 \epsilon_{0} V_{c}\right)} e^{i \mathbf{k} \cdot \mathbf{r}_{j}} \sin \left(m \pi z_{j} / L_{z}\right)$ ( $\epsilon_{0}$ is the electrical permittivity of free space, $V_{c}$ is the cavity quantization volume and $z_{j}$ is the position of the molecule along the cavity longitudinal axis), $\bar{f}$ denotes the complex conjugate of $f$, and $H_{\mathrm{P}^{2}}$ is the molecular self-polarization energy [59]. Although this term ensures the existence of a ground-state for the composite system [60] and it becomes essential for an appropriate treatment of a system with total light-matter interaction energy approaching or surpassing the bare cavity and molecular frequencies [61], $H_{\mathrm{P}^{2}}$ can be neglected under the strong coupling conditions assumed here. Therefore, we will disregard this term onward.

The length scale over which the cavity mode profile varies substantially (of order $0.1-20 \mu \mathrm{m}$ ) is much larger than typical molecular diameters (of order $0.5-5 \mathrm{~nm}$ ). Thus, under strong coupling, the $\mathbf{k} \approx 0$ cavity modes interact coherently with material polarization consisting of a macroscopic number of molecules. This notion forms the basis for neglecting spatial, orientational and energetic dispersion of the molecular excitations, since fluctuations of these quantities are necessarily weak effects compared to the collective light-matter interactions from which polaritons emerge. Fluctuations about the mean values of the molecular transition frequency and dipole moment can lead to dephasing-induced polariton decay $[62,63]$, weak-coupling of light to states which are dark according to Eq. 3, as well as polariton [64, 65] and dark-state localization $[64,66]$. Since we are not concerned with transport phenomena, we will not include them in our model, although Sec. 4 qualitatively analyzes their implications to our main results. Despite neglecting these effects, we highlight that our input-output treatment of the material and photonic components naturally accounts for polariton dissipation via cavity leakage and molecular homogeneous dephasing [43, 54, 55, 67]. In Eq. 3, we also assumed validity of the so-called rotatingwave-approximation: only light-matter interactions preserving the total number of cavity and molecular excitations are retained. This approximation is justified by $\sqrt{\sum_{i=1}^{N}\left|g_{i \mathbf{k}}\right|^{2}} \ll \omega_{0}, \quad \forall \mathbf{k}$.

We aim to investigate the nonlinear response of the hybrid system to an input radiation field with $\mathbf{k} \in \mathbb{R}^{2}$ centered at $\mathbf{k}_{0} \approx 0$, with a small width $\delta \mathbf{k}$. The frequency $\omega_{\mathbf{k}_{0}}$ is nearly resonant with the bare molecule fundamental frequency $\omega_{0}$. Therefore, we shall retain only a single cavity-mode corresponding to $\mathbf{k}_{0} \approx 0$. This assumes there is no variation in the polariton nonlinear response with respect to changes of magnitude $|\delta \mathbf{k}|$ in the incident wave-vector $\mathbf{k}_{0}$.

Based on the above, we simplify $H_{L}(t)$ and $H_{\mathrm{LM}}$ and employ the following effective Hamiltonian for the hybrid cavity-matter system

$$
\begin{aligned}
H_{T}(t)= & \hbar \omega_{c} b^{\dagger} b+\sum_{i=1}^{N} \hbar \omega_{0} a_{i}^{\dagger} a_{i}-\hbar \Delta \sum_{i=1}^{N} a_{i}^{\dagger} a_{i}^{\dagger} a_{i} a_{i} \\
& -\sum_{i=1}^{N} \mu\left(E_{0}^{c} a_{i}^{\dagger} b+\bar{E}_{0}^{c} b^{\dagger} a_{i}\right) \\
& -i \hbar \sqrt{\frac{\kappa}{2}}\left\{\left[b_{\text {in }}^{\mathrm{L}}(t)\right]^{\dagger} b-b^{\dagger} b_{\text {in }}^{\mathrm{L}}(t)\right\},
\end{aligned}
$$

where $\omega_{c} \equiv \omega_{\mathbf{k}_{0}}, b=b_{\mathbf{k}_{0}}, b_{\mathbf{k}_{0} \text { in }}^{\mathrm{L}}=b_{\text {in }}^{\mathrm{L}}$, and $\mu E_{0}^{c} \equiv g_{j \mathbf{k}_{0}}=$ $i \mu \sqrt{\hbar \omega_{c} /\left(2 \epsilon_{0} V_{c}\right)}$.

\subsection{Nonlinear molecular polarization under strong light-matter coupling}

The optical response of a hybrid microcavity can be investigated by measuring the transmission, reflection or absorption spectrum of light input into the system. For instance, transmission and reflection spectra can be obtained by applying the input-output relations to the steady-state cavity field $b(t)=\sum_{\omega>0} b(\omega) e^{-i \omega t}$. Because the cavity is weakly-coupled to the external fields, the expectation value $\langle b(t)\rangle$ admits a perturbative expansion in powers of the input amplitude $\langle b(t)\rangle=\sum_{p=1}^{\infty}\langle b(t)\rangle^{(2 p-1)}$, where $\langle b(t)\rangle^{(2 p-1)}=O\left[\left|b_{\mathrm{in}}^{\mathrm{L}}\right|^{2 p-1}\right]$ (only odd powers of the input field appear in the cavity response because the material is assumed homogeneous and symmetric with respect to spatial inversion $[1,12])$. The material polarization $P(t)=\mu \sum_{i=1}^{N} a_{i}(t)$ is strongly coupled to the optical cavity. Therefore, molecular observables also admit a perturbative expansion in powers of $\left|b_{\mathrm{in}}^{\mathrm{L}}\right|$. Note that the empty cavity is a linear system, and thus, the source of the nonlinear part of $\langle b(t)\rangle$ is the molecular subsystem (specifically, the source of $\langle b(t)\rangle^{(3)}$ is $\langle P(t)\rangle^{(3)}=\sum_{i=1}^{N} \mu\left\langle a_{i}(t)\right\rangle^{(3)}$; see SI Sec. 2). Therefore, $\langle P(t)\rangle^{(3)}$ directly determines the amplitude of the nonlinear optical response of a strongly coupled system as measured by the output transmitted and reflected light.

Neglecting quantum fluctuations of the input field, $b_{\text {in }}(t)$ is a complex number that we express as:

$$
b_{\mathrm{in}}(t)=i \sum_{\omega>0} \sqrt{\frac{\mathcal{P}(\omega)}{\hbar \omega}} e^{i \theta_{\mathrm{in}}(\omega)} e^{-i \omega t},
$$


where $\mathcal{P}(\omega)$ is the power of the free space mode with frequency $\omega$ driving the cavity, and $\theta_{\text {in }}(\omega)$ is its phase (SI Sec. 1).

It follows (SI Sec. 2) that the third-order polarization in the frequency domain $\langle P\rangle^{(3)}\left(\omega_{s}\right)$ can be written in terms of the input electric fields as follows

$$
\begin{aligned}
\langle P\rangle^{(3)}\left(\omega_{s}\right)= & \sum_{\omega_{u}, \omega_{v}, \omega_{w}} \chi^{(3)}\left(-\omega_{s} ; \omega_{v},-\omega_{w}, \omega_{u}\right) E_{\mathrm{in}}^{(+)}\left(\omega_{v}\right) \times \\
& E_{\mathrm{in}}^{(-)}\left(\omega_{w}\right) E_{\mathrm{in}}^{(+)}\left(\omega_{u}\right)+\text { h.c. }
\end{aligned}
$$

where $\omega_{s}>0$ is the signal frequency, the brackets denote expectation values, the driving frequencies $\omega_{u}, \omega_{v}, \omega_{w}$ are all positive, the input fields $E_{\text {in }}^{(+)}\left(\omega_{u}\right)$ are directly proportional to the $b_{\text {in }}\left(\omega_{u}\right)$ (see SI Sec. 1), and $\chi^{(3)}$ is the nonlinear molecular susceptibility [12] under strong lightmatter interaction conditions. The ratio between $\chi^{(3)}$ and the bare molecular system nonlinear susceptibility $\chi_{0}^{(3)}$ provides an external-field independent measure of strong light-matter coupling effects on the optical nonlinearities of an arbitrary molecular system.

To obtain $\chi^{(3)}[1]$ for the system described by Eq. 4, we solve perturbatively the Heisenberg-Langevin equations of motion (EOM) for the molecular polarization to thirdorder in the driving field $b_{\text {in }}^{\mathrm{L}}[68-70]$. The EOMs for the cavity and material polarization expectation values admit simple solutions since the initial condition (groundstate) and the time-evolution of our system ensures that it remains in a pure state at all times. The result is (SI Sec. 2):

$$
\begin{aligned}
\chi^{(3)}\left(-\omega_{s} ; \omega_{v},-\omega_{w}, \omega_{u}\right)= & N \mu(2 \hbar \Delta) G_{m m}\left(\omega_{s}\right) \bar{G}_{m m}\left(\omega_{w}\right) \Gamma_{m m, m m}\left(\omega_{u}+\omega_{v}\right) G_{m m}\left(\omega_{u}\right) G_{m m}\left(\omega_{v}\right) \times \\
& {\left[\mu \sqrt{\frac{2 \mathcal{F}}{\pi}} G_{p p}^{(0)}\left(\omega_{v}\right) \frac{\hbar \kappa}{2}\right]\left[\mu \sqrt{\frac{2 \mathcal{F}}{\pi}} \frac{\hbar \kappa}{2} \bar{G}_{p p}^{(0)}\left(\omega_{w}\right)\right]\left[\mu \frac{\hbar \kappa}{2} \sqrt{\frac{2 \mathcal{F}}{\pi}} G_{p p}^{(0)}\left(\omega_{u}\right)\right] \delta_{\omega_{s}, \omega_{v}-\omega_{w}+\omega_{u}}, }
\end{aligned}
$$

where $\mathcal{F}$ is the cavity finesse (the electromagnetic field intensity in a resonant cavity is stronger than in free space by the factor $2 \mathcal{F} / \pi$, or alternatively, $\mathcal{F}=Q / m$, where $m$ is the aforementioned band index, and $Q=\omega_{c} / \kappa$ is the quality factor; SI Sec. I and Ref. [46]), and $G_{m m}(\omega)$ is the Fourier transform (FT) of the retarded singlemolecule propagator

$$
G_{m m}(\omega)=\frac{1}{\hbar \omega-\hbar \omega_{0}+i \hbar \gamma_{m} / 2-\frac{\left|\mu E_{0}\right|^{2} N}{\hbar \omega-\hbar \omega_{c}+i \kappa / 2}}
$$

Note the real part of the poles of $G_{m m}(\omega)$ are the fundamental polariton resonance frequencies

$$
\begin{aligned}
& \omega_{\mathrm{LP}}=\frac{\omega_{c}+\omega_{0}}{2}-\frac{\sqrt{\left(\omega_{c}-\omega_{0}\right)^{2}+\Omega_{R}^{2}}}{2} \\
& \omega_{\mathrm{UP}}=\frac{\omega_{c}+\omega_{0}}{2}+\frac{\sqrt{\left(\omega_{c}-\omega_{0}\right)^{2}+\Omega_{R}^{2}}}{2} .
\end{aligned}
$$

where $\Omega_{R}=2\left|\mu E_{0}^{c}\right| \sqrt{N} / \hbar$ is the Rabi frequency (splitting). The imaginary parts of the polariton poles in $G_{m m}(\omega)$ correspond to their (linear) absorption linewidths. Under weak-coupling conditions, we can neglect the cavity-induced self-energy $\frac{\left|\mu E_{0}\right|^{2} N}{\hbar \omega-\hbar \omega_{c}+i \kappa / 2}$ to obtain the bare molecule propagator $G_{m m}^{(0)}(\omega)=1 /(\hbar \omega-$ $\left.\hbar \omega_{0}+i \hbar \gamma_{m} / 2\right)$. Similarly, the photon-photon correlator $G_{p p}(\omega)$ has resonances at the polariton frequencies, as is clear from its explicit form:

$$
G_{p p}(\omega)=\frac{1}{\hbar \omega-\hbar \omega_{c}+i \hbar \kappa / 2-\frac{\left|\mu E_{0}^{c}\right|^{2} N}{\hbar \omega-\hbar \omega_{0}+i \hbar \gamma_{m} / 2}} .
$$

In the weak-coupling limit, $G_{p p}(\omega)$ approaches the empty cavity frequency-domain propagator $G_{p p}^{(0)}(\omega)=1 /(\hbar \omega-$ $\left.\hbar \omega_{c}+i \hbar \kappa / 2\right)$.

The function $\Gamma_{m m, m m}\left(\omega_{u}+\omega_{v}\right)$ is the two-particle elastic scattering matrix element given by:

$$
\Gamma_{m m, m m}(\omega)=\frac{\left(\hbar \omega-2 \hbar \tilde{\omega}_{0}\right)\left(\hbar \omega-\hbar \tilde{\omega}_{0}-\hbar \tilde{\omega}_{c}\right)\left[\left(\hbar \omega-2 \hbar \tilde{\omega}_{0}\right)\left(\hbar \omega-2 \hbar \tilde{\omega}_{c}\right)-4 g^{2} N\right]}{D(\omega)}
$$

where $D(\omega)$ is a 4 th-order polynomial of $\omega$ given by:

$$
\begin{aligned}
D(\omega)= & D^{(0)}(\omega)-2 g^{2} N\left(\hbar \omega-2 \hbar \tilde{\omega}_{0}\right)\left(\hbar \omega-2 \hbar \tilde{\omega}_{0}+2 \hbar \Delta\right) \\
& -2 g^{2}(N-1)\left(\hbar \omega-2 \hbar \tilde{\omega}_{0}+2 \hbar \Delta\right)\left(\hbar \omega-2 \hbar \tilde{\omega}_{c}\right) \\
& -2 g^{2}\left(\hbar \omega-2 \hbar \tilde{\omega}_{c}\right)\left(\hbar \omega-2 \hbar \tilde{\omega}_{0}\right),
\end{aligned}
$$

where $g=\left|\mu E_{0}^{c}\right|$ is the single-molecule light-matter coupling and $D^{(0)}(\omega)=\left(\hbar \omega-\hbar \tilde{\omega}_{c}-\hbar \tilde{\omega}_{0}\right)\left(\hbar \omega-2 \hbar \tilde{\omega}_{0}+2 \hbar \Delta\right) \times$ $\left(\hbar \omega-2 \hbar \tilde{\omega}_{c}\right)\left(\hbar \omega-2 \hbar \tilde{\omega}_{0}\right)$. The roots of $D(\omega)$ correspond to the bright two-particle resonances of the hybrid system, as can be verified by comparison to the eigenval- 


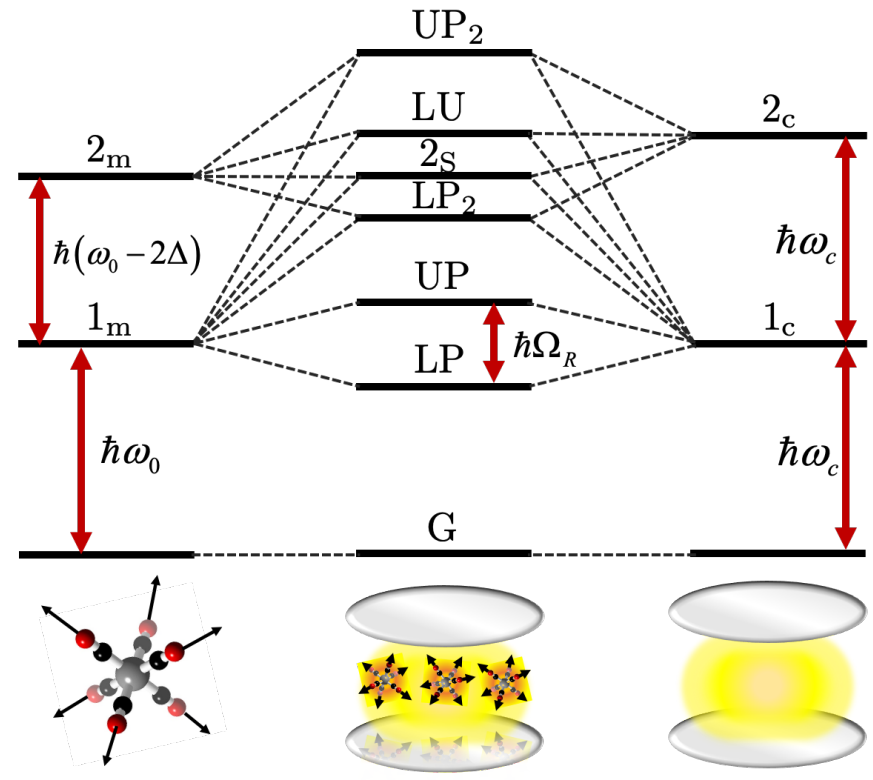

FIG. 2. Energy level diagram for a model system with zero-detuning $\left(\omega_{c}=\omega_{0}\right)$ and Rabi splitting $\Omega_{R}>$ anharmonicity $2 \Delta$, including only bright excitations of the single and two-polariton manifolds (SI Sec. 7).

ues of the doubly-excited block of the Hamiltonian in Eq. 4 with $b_{\text {in }}^{\mathrm{L}}=0$ (see SI Sec. 7 for a discussion and analytical results for the $\omega_{c}=\omega_{0}$ case). In the large $N$ limit appropriate to almost all experimental studies of infrared strong coupling, the real parts of the twoparticle resonances in the zero-detuning case are given by $\omega_{2 \mathrm{UP}}=2 \omega_{\mathrm{UP}}+O(g / \sqrt{N}), \omega_{\mathrm{LU}}=\omega_{c}+\omega_{0}, \omega_{2_{m}}=$ $2 \omega_{0}-2 \Delta+O(\Delta / N)$, and $\omega_{2 \mathrm{LP}}=2 \omega_{\mathrm{LP}}+O(g / \sqrt{N})$, where the subscripts label the dominant character of each state, e.g., the highest-frequency resonance is dominated by the component with a doubly-excited UP mode while the resonance with frequency $\omega_{\mathrm{LU}}$ corresponds to that containing an LP,UP pair (see Fig. 2).

Physically, $\Gamma_{m m}\left(\omega_{u}+\omega_{v}\right) \propto G_{m m, m m}\left(\omega_{u}, \omega_{v}\right)$ (SI Sec. $2)$, where $G_{m m, m m}(\omega)$ is the frequency-domain singlemolecule two-excitation propagator, i.e., the FT of the probability amplitude that a molecule initially in its doubly excited-state remains in the same state after time $t$.

Importantly, when $N \rightarrow \infty$, Eq. 12 becomes:

$$
\Gamma_{m m, m m}(\omega) \approx \Gamma_{m m, m m}^{(0)}(\omega) \equiv \frac{\omega-2 \tilde{\omega}_{0}}{\omega-2 \tilde{\omega}_{0}+2 \Delta}, N \rightarrow \infty
$$

where $\Gamma^{(0)}(\omega)$ is the bare single-molecule two-particle (elastic) scattering matrix (see next subsection and SI Sec. 4). This result is expected, since $\Gamma_{m m, m m}(t)$ describes the time-dependent propagation of singlemolecule doubly excited-states under interaction with the optical cavity, and as we show in SI Sec. 7, the totally-symmetric doubly-excited molecular state $\left|2_{m}\right\rangle=$ $\frac{1}{\sqrt{N}} \sum_{i=1}^{N}\left|2_{i}\right\rangle$ (where $\left|2_{i}\right\rangle$ is the state where the $i$ th molecule is in the 2nd excited-state while the cavity and all other molecules are in the ground-state) is only weakly-coupled to two-polariton states via an interaction that is proportional to the single-molecule light-matter coupling $g$. Therefore, while polaritons play an essential role as intermediate states for TPA by the molecular subsystem, Eq. 14 indicates the dynamics of molecular doubly excited-states is almost insensitive to their coupling to the cavity electromagnetic field in the ensemble strong coupling limit.

To gain further insight into the molecular nonlinear polarization in the strong light-matter coupling regime, we now compare Eq. 7 to the nonlinear susceptibility of the bare molecules in free space (under the rotating-wave approximation) given by

$$
\chi_{0}^{(3)}\left(-\omega_{s} ; \omega_{v},-\omega_{w}, \omega_{u}\right)=N \mu(2 \hbar \Delta) \mu^{3} G_{m m}^{(0)}\left(\omega_{s}\right) \bar{G}_{m m}^{(0)}\left(\omega_{w}\right) \Gamma_{m m, m m}^{(0)}\left(\omega_{u}+\omega_{v}\right) G_{m m}^{(0)}\left(\omega_{v}\right) G_{m m}^{(0)}\left(\omega_{u}\right) \delta_{\omega_{s}, \omega_{v}-\omega_{w}+\omega_{u}}
$$

By contrasting Eqs. 15 and 7 , we find that the nonlinear optical response of a molecular system (e.g., solution [37, 71], polymer [72, 73], etc) in an optical microcavity is significantly distinct from that in free space mainly because of: (i) near-resonant intracavity field intensity enhancement [which renormalizes the induced molecular transition dipole moments $\left.\mu \rightarrow \tilde{\mu}(\omega)=\mu \sqrt{2 \mathcal{F} / \pi} i \hbar \sqrt{\kappa / 2} G_{p p}^{(0)}(\omega)\right]$, and (ii) the appearance of new (polariton) resonances corresponding to hybrid superpositions of molecular polarization and cavity modes. In other words, the molecular nonlinear response under strong coupling can be written entirely in terms of cavity-renormalized single-particle
$\left[G_{m m}^{(0)}(\omega) \rightarrow G_{m m}(\omega)\right]$ and two-particle molecular response functions $\left[\Gamma_{m m, m m}^{(0)}(\omega) \rightarrow \Gamma_{m m, m m}(\omega)\right]$ which are non-perturbatively dressed by the interaction with the cavity field, as well as (ii) molecular transition dipoles $\mu$ which are renormalized by factors that depend on the cavity finesse $\mathcal{F}$ and the bare photon propagator $[\mu \rightarrow \tilde{\mu}(\omega)]$. The renormalization of the induced molecular dipoles is a result of the well-known enhancement of the intracavity electric field relative to free space $[46,74]$. In the weak coupling regime, a Purcell-like result follows where the molecular nonlinear susceptibility in a microcavity (Eq. 7) is simply related to that of the bare system (Eq. 15), $\chi^{(3)} \rightarrow \chi_{0}^{(3)} \times$ intracavity field enhancement 
factors.

As discussed in Sec. 3, by virtue of the cavitymatter strong coupling, the nonlinear polarization contribution to the energy absorbed by the molecular subsystem is not directly proportional to the imaginary part of $\chi^{(3)}(-\omega ; \omega,-\omega, \omega)$ (in contrast to the nonlinear absorption of bare molecules; see SI Sec. 5). Therefore, we provide additional comments and a numerical comparison of the real and imaginary parts of Eqs. 7 and 15 as a function of cavity detuning and Rabi splitting in SI Sec. 6 , and focus below on the nonlinear absorption spectrum of the strongly coupled material.

\section{POLARITON-ENHANCED TWO-PHOTON ABSORPTION}

The steady-state rate of excitation of a molecular system driven by the electromagnetic field can be written as (SI Secs. 3 and 5)

$$
W_{T}(\omega) \equiv \frac{2}{\hbar}\left[\operatorname{Im}\left\langle[E(\omega)]^{\dagger} P(\omega)\right\rangle\right]
$$

where $E(\omega)$ is the frequency-domain representation of the free space or cavity Heisenberg electric field operator. For a molecular system in free space interacting weakly with a classical monochromatic EM field with (positivefrequency) amplitude $E_{\text {in }}^{(+)}(\omega)$, it follows that the photon absorption rate (in the rotating-wave approximation) is [1]

$$
\begin{aligned}
W_{0}(\omega) \equiv & \frac{2}{\hbar} \operatorname{Im}\left[\chi_{0}^{(1)}(-\omega ; \omega)\right]\left|E_{\mathrm{in}}^{(+)}(\omega)\right|^{2} \\
& +\frac{2}{\hbar} \operatorname{Im}\left[\chi_{0}^{(3)}(-\omega ; \omega,-\omega, \omega)\right]\left|E_{\mathrm{in}}^{(+)}(\omega)\right|^{4}+\ldots
\end{aligned}
$$

This expression is clearly invalid when the molecular ensemble interacts strongly with a cavity, since in this instance, the cavity field and the material electrical polarization are correlated, and therefore $\langle E(\omega) P(\omega)\rangle$ cannot (in general) be factorized into $\langle E\rangle(\omega)\langle P\rangle(\omega)$ (where $E$ refers to the cavity EM field). Nevertheless, the external input field interacts weakly with the cavity, and the rate of absorption by the strongly coupled molecular system admits the following perturbative expansion in powers of the input field amplitude

$W(\omega)=\frac{2}{\hbar} \operatorname{Im}\left[\left\langle\left[E_{c}(\omega)\right]^{\dagger} P(\omega)\right\rangle^{(2)}+\left\langle\left[E_{c}(\omega)\right]^{\dagger} P(\omega)\right\rangle^{(4)}\right]+\ldots$

where $E_{c}(\omega)=E_{0}^{c} b(\omega)$. The contribution to the absorption spectrum dependent on the nonlinear response of the molecular subsystem is given by $W^{\mathrm{NL}}(\omega)=$ $\frac{2}{\hbar} \operatorname{Im}\left[\left\langle E_{c}^{\dagger}(\omega) P(\omega)\right\rangle^{(4)}\right]$. In SI Sec. 3, we obtain $W^{\mathrm{NL}}(\omega)$ by employing the Heisenberg-Langevin EOMs following the same approach taken to derive Eq. 7.
For simplicity, we restrict our analysis of the nonlinear absorption spectrum to the zero-detuning case where $\omega_{c}=\omega_{0}$. We also simplify $W^{\mathrm{NL}}(\omega)$ by using the following conditions necessarily valid at strong coupling: $\Omega_{R} \gg$ $\hbar \eta_{s} \equiv \hbar\left(\kappa+\gamma_{m}\right)$, and $\Omega_{R} \gg \hbar \eta \equiv \hbar \kappa \gamma_{m} /\left(\kappa+\gamma_{m}\right)$. Under these conditions, the nonlinear component of molecular absorption under strong coupling with a cavity can be expressed as $W^{\mathrm{NL}}(\omega)=\sum_{\alpha=1}^{4} W^{\mathrm{NL}_{\alpha}}(\omega)\left|E_{\text {in }}^{(+)}(\omega)\right|^{4}$, where

$$
\begin{aligned}
W^{\mathrm{NL}_{1}}(\omega) \approx & -\frac{2 \eta \kappa}{\hbar}\left[\frac{1}{4\left(\omega-\omega_{0}\right)^{2}+\kappa^{2}}+\frac{1}{\left(\Omega_{R} / \hbar\right)^{2}}\right] \\
& \times \operatorname{Re}\left[\sqrt{\frac{2 \mathcal{F}}{\pi}} \chi^{(3)}(\omega)\right]
\end{aligned}
$$

$$
\begin{aligned}
& W^{\mathrm{NL}_{2}}(\omega) \approx \frac{4 \eta}{\hbar} \frac{\omega-\omega_{0}}{4\left(\omega-\omega_{0}\right)^{2}+\kappa^{2}} \operatorname{Im}\left[\sqrt{\frac{2 \mathcal{F}}{\pi}} \chi^{(3)}(\omega)\right] \\
& W^{\mathrm{NL}_{3}}(\omega) \approx \eta \frac{4 \Delta^{2} N}{\left(\omega-\omega_{0}\right)^{2}+\gamma_{m}^{2} / 4} \frac{\left|\left\langle a_{i} a_{i}\right\rangle^{(2)}(2 \omega)\right|^{2}}{\left|E_{\text {in }}^{(+)}(\omega)\right|^{4}}, \\
& W^{\mathrm{NL}_{4}}(\omega) \approx-\eta \frac{\left(2 \omega-\omega_{20}\right) 2 \Delta N}{\left(\Omega_{R} / 2 \hbar\right)^{2}} \frac{\left|\left\langle a_{i} a_{i}\right\rangle^{(2)}(2 \omega)\right|^{2}}{\left|E_{\text {in }}^{(+)}(\omega)\right|^{4}},
\end{aligned}
$$

where $\chi^{(3)}(\omega) \equiv 6 \chi^{(3)}(-\omega ; \omega,-\omega, \omega), \omega_{20} \equiv 2 \omega_{0}-2 \Delta$, and

$$
\begin{aligned}
\left\langle a_{i} a_{i}\right\rangle^{(2)}(2 \omega) & =-\Gamma_{m m, m m}(2 \omega) G_{m m}(\omega) G_{m m}(\omega) \\
& \times\left[\mu \sqrt{\frac{2 \mathcal{F}}{\pi}} G_{p p}^{(0)}(\omega) \frac{\hbar \kappa}{2} E_{\mathrm{in}}^{(+)}(\omega)\right]^{2} .
\end{aligned}
$$

The expression for the nonlinear absorption by the molecular system under strong coupling with a cavity is more complicated relative to the bare system given by $W_{0}^{\mathrm{NL}}(\omega)\left|E_{\text {in }}^{(+)}(\omega)\right|^{4}=$ $\frac{2}{\hbar} \operatorname{Im}\left[\chi_{0}^{(3)}(-\omega ; \omega,-\omega, \omega)\right]\left|E_{\text {in }}^{(+)}(\omega)\right|^{4}$. For example, $W^{\mathrm{NL}}(\omega)$ shows dependence on both the real and imaginary parts of $\chi^{(3)}$ (Eqs. 19 and 20) in addition to the steady-state population of molecular doubly excitedstates $P_{2 m}^{T}(\omega)=\sum_{i=1}^{N}\left|\left\langle a_{i} a_{i}\right\rangle^{(2)}(2 \omega)\right|^{2} / 2$ (Eqs. 21 and $22)$. This additional complexity of nonlinear absorption under strong coupling conditions is expected, since while external fields acting on the bare system drives transitions between three molecular states (ground, first and second excited-state), at least seven energy levels (Fig. 2) may play a role in the nonlinear response of a material strongly coupled to an optical cavity. Nevertheless, the main features of $W^{\mathrm{NL}}(\omega)$ can be obtained from Eqs. 19-22. Specifically,

1. The nonlinear absorption intensity is largest when the input field is nearly-resonant with either the LP or UP, since this maximizes $\left|G_{m m}(\omega)\right|^{4}$ which 


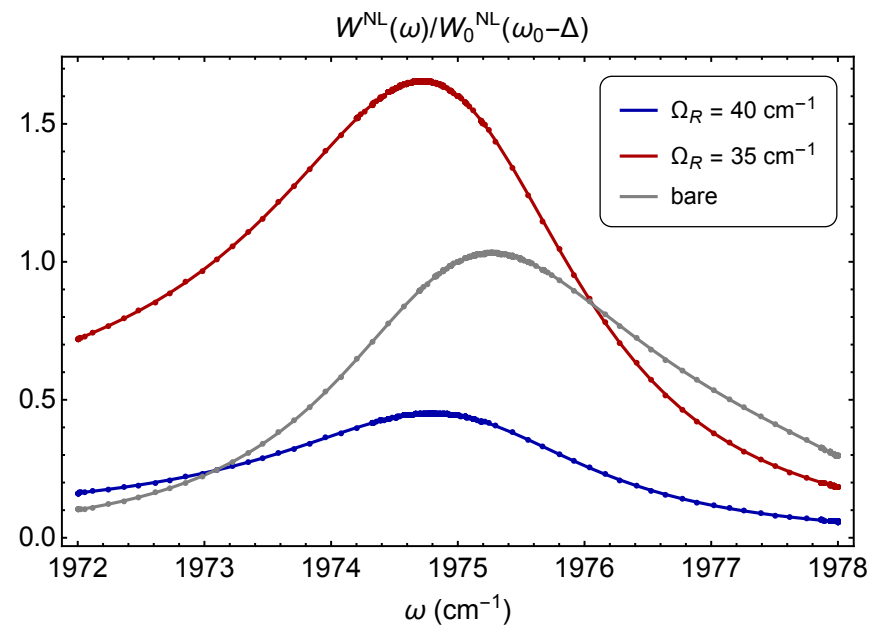

FIG. 3. Ratio of two-photon absorption rate of two strongly coupled $\left(\omega_{0}=\omega_{c}=1983 \mathrm{~cm}^{-1}, \gamma_{m}=\kappa=3 \mathrm{~cm}^{-1}, \Delta=\right.$ $8 \mathrm{~cm}^{-1}$, and $\Omega_{R}=40 \mathrm{~cm}^{-1}$ or $\Omega_{R}=35 \mathrm{~cm}^{-1}$ ) systems relative to that of the molecular ensemble in free space normalized by the maximum of the latter.

appears in all of Eqs. 19-22. Physically, the polariton resonance condition for maximal photon absorption is a consequence of the optical filtering performed by a microcavity (off-resonant external fields are suppressed relative to the resonant).

2. $W^{\mathrm{NL}_{3}}(\omega)$ is the only component of $W^{\mathrm{NL}}(\omega)$ which is positive for all values of the input frequency. Therefore, it necessarily gives molecular excitedstate absorption contributions to $W^{\mathrm{NL}}(\omega)$. Further evidence is given by the fact that $W^{\mathrm{NL}_{3}}(\omega)$ is proportional to the steady-state population of molecules in the doubly-excited state, and thus,

$$
W^{\mathrm{NL}_{3}}(\omega) \approx \frac{8 \eta \Delta^{2}}{\left(\omega-\omega_{0}\right)^{2}+\gamma_{m}^{2} / 4} \frac{P_{2_{m}}^{\mathrm{T}}(2 \omega)}{\left|E_{\mathrm{in}}^{(+)}(\omega)\right|^{4}} .
$$

All other contributions to the nonlinear absorption can be either positive (when excited-state absorption processes dominate) or negative (when ground-state bleach and stimulated emission processes dominate [1]) depending on $\omega$.

3. Based on items 1 and 2, we expect the TPA rate will be largely enhanced relative to free space when the doubly-excited molecular states are approximately resonant with either one of the available two-polariton transitions (see Fig. 1), i.e.,

$$
\begin{aligned}
& 2 \omega_{0}-2 \Delta=2 \omega_{\mathrm{LP}}, \quad \Delta>0, \text { or } \\
& 2 \omega_{0}-2 \Delta=2 \omega_{\mathrm{UP}}, \quad \Delta<0,
\end{aligned}
$$

since in this case, all response functions showing up in Eqs. 7 and $19-23$ (namely, $G_{m m}(\omega)$, and the scattering amplitude $\left.\Gamma_{m m, m m}(2 \omega)\right)$ become resonant at $\omega=\omega_{\mathrm{LP}}$ (if $\Delta>0$ ) or $\omega=\omega_{\mathrm{UP}}$ (if $\Delta<0$ ).
Physically, the polaritons provide the resonant optical window to efficiently drive the transitions of interest. In the studied case of zero cavity detuning, the conditions described in Eq. 25 can be summarized as the Rabi splitting being equal to the anharmonic shift, that is, $\Omega_{R}= \pm 2 \Delta$.

When the criteria in Eq. 25 are satisfied, we expect strong enhancement of nonlinear absorption based on the following argument: if the input field consists of photons with $\omega=\omega_{0}-\Delta$ and Eq. 25 is satisfied, polaritons will be efficiently pumped, and a fraction of those will subsequently decay by populating molecular doubly-excited states. In other words, when the two-polariton resonance condition is satisfied, the molecular doubly excited-states provides an efficient sink for the energy stored in two-polariton modes. This effect was indeed reported in a recent experiment [42], where evidence was given that (for systems with weak system-bath interactions and slow molecular polarization dephasing) the second excited vibrational state was preferentially populated over the first when the pump (input) field was resonant with LP.

4. Conversely, in the limit where two-polariton states are highly off-resonant with the molecular TPA $\left(\left|2 \Delta-\Omega_{R}\right| \gg 0\right.$, Fig. 3), the nonlinear response substantially weakens. In this limit, the studied model approaches the Tavis-Cummings [75], where a collection of two-level systems interact strongly with a single-mode cavity. The nonlinear response given by this system is known to become negligible in the large $N$ limit [76] (e.g., as we show in the SI Sec. 7, in the Tavis-Cummings model, the twolevel system nonlinearity produces a large $N$ limit anharmonic shift proportional to $\left.\left|\mu E_{0}^{c}\right| / \sqrt{N}\right)$.

It follows, therefore, that the condition given in Eq. 25 allows the harnessing of the enhanced electromagnetic field of optical cavities to enhance TPA.

Points 3 and 4 are the main conclusions of our work. We will now quantitatively illustrate that under experimentally accessible conditions, it is possible to employ cavity-strong coupling to substantially enhance the TPA cross section of a resonant molecular system.

In Fig. 3, we present the infrared TPA spectrum for a molecular system in free space (we take representative parameters for $\mathrm{W}(\mathrm{CO})_{6}$ in solution [37, 42], $\left.\omega_{0}=1983 \mathrm{~cm}^{-1}, \Delta=8 \mathrm{~cm}^{-1}, \gamma=3 \mathrm{~cm}^{-1}\right)$, and under strong coupling with a microcavity $\left(\omega_{c}=\omega_{0}, \kappa=\gamma\right)$ for $\Omega_{R}=40 \mathrm{~cm}^{-1}$ and $\Omega_{R}=35 \mathrm{~cm}^{-1}$. The curves are normalized by the maximum of the bare system TPA. Figure 3 shows the strong dependence of the TPA cross-section on the light-matter interaction: when $\Omega_{R}=40 \mathrm{~cm}^{-1}$ $\left(\Omega_{R}-2 \Delta=24 \mathrm{~cm}^{-1}\right)$, the nonlinear absorption is suppressed relative to that given by the bare system. However, a slight decrease of $\Omega_{R}$ to $35 \mathrm{~cm}^{-1}$ leads to enhanced TPA due to a stronger spectral overlap between the $\mathrm{LP}_{2}$ 


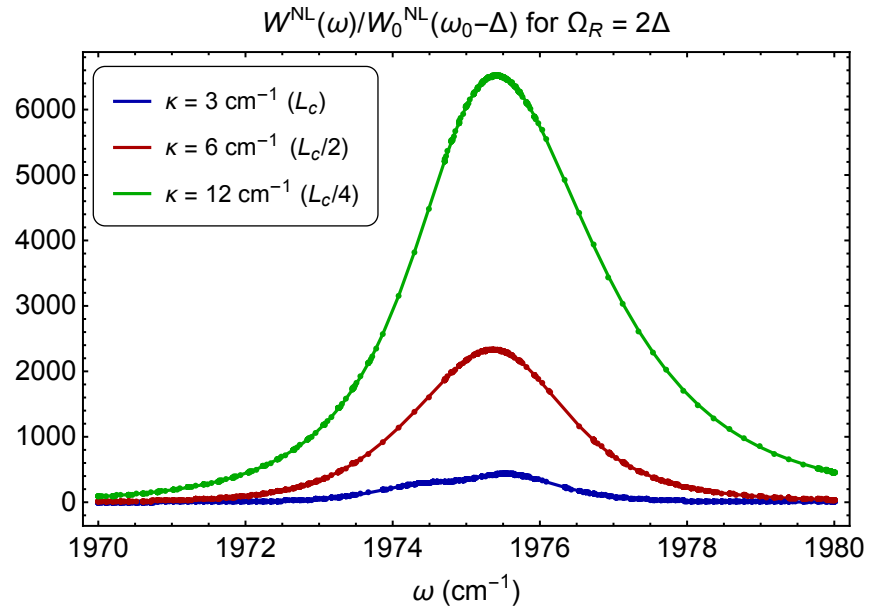

FIG. 4. Enhancement of TPA rate of strongly coupled system when $\Omega_{R}=2 \Delta$, with $\omega_{0}=\omega_{c}, \gamma=3 \mathrm{~cm}^{-1}, \Delta=$ $8 \mathrm{~cm}^{-1}$ for optical microcavities with different cavity lengths $\left(L_{c}, L_{c} / 2, L_{c} / 4\right)$ and corresponding decay rates.

mode and the molecular doubly excited-state transition from the ground-state.

In Fig. 4, we explore the great potential for obtaining polariton-enhanced TPA with optical microcavities of varying longitudinal lengths $L_{c}, L_{c} / 2$ and $L_{c} / 4$ (with $L_{c}=10 \mu m$, and cavity-mode indices $m=4, m=2$ and $m=1$, respectively, which would require cavity mirrors with transmissivity $|t|^{2} \approx 0.01 \%$ ). We assume the cavities are resonant with the molecular fundamental transition, and the TPA condition $\Omega_{R}=2 \Delta=16 \mathrm{~cm}^{-1}$ is valid (the remaining bare molecule parameters are the same as in Fig. 3). The two main conclusions from Fig. 4 are that: (a) the polariton-mediated TPA cross section predicted by our model can be larger than the bare one by close to 4 orders of magnitude for accessible parameters, and (b) a decrease in cavity length leads to stronger nonlinear signals, so that the cavity-mediated TPA will be maximally efficient when the strongly coupled cavity mode has the lowest possible longitudinal quantum number and mirrors with highest available reflectivity. These conditions, in fact, maximize the intracavity electromagnetic field enhancement relative to free space (see SI Sec. $1)$.

A similar increase in nonlinear response signal strength with decreasing molecular concentration (Fig. 3) or cavity longitudinal length (Fig. 4), was observed and qualitatively analyzed in a different context in Ref. [77].

\section{DISCUSSION AND CONCLUSIONS}

The computed enhanced polariton-mediated TPA provides an upper bound estimate to future measurements of TPA under strong coupling conditions. Experiments performed on analogous systems could give reduced enhancements relative to those presented here for at least two reasons: (a) the intracavity enhancement factor (represented by the cavity finesse) varies spatially according to the cavity mode profile $(\sin (\pi z / L)$ in the simplest case), whereas we assumed that all molecules are within a small region around an antinode of the cavity field (so that the cavity field enhancement factor is maximal), and (b) inhomogeneous broadening of the molecular subsystem which allows for potentially fast polariton relaxation into reservoir (dark) modes, as well as reduction in efficiently of polariton pumping due to photonic intensity borrowing. Although we recognize the importance of these approximations, we disregard them in our explorations, since the inhomogeneity of the cavity mode profile is expected to change the nonlinear response properties by factors of order 1 (alternatively, spacers may be introduced between the molecular system and the optical cavity so that the molecules occupy only a small region around the antinode of the cavity mode profile), while (lower) polariton decay can be slowed down by increasing the Rabi splitting and (or) lowering the temperature. Moreover, polariton transitions are well-known to be homogeneously broadened within their lifetimes [47], and therefore, for molecular systems with significant inhomogeneously broadened transitions, we expect polariton lineshapes to be significantly narrower than that of the bare system (given the polariton "hole-burning" effect [78] yielding subnatural linewidths). In this instance, the mechanism for polariton-mediated TPA presented in our article would become even more efficient than in the model considered here.

While our study focused on infrared polaritonics, we note that the phenomenology observed in molecular vibrational and electronic strong coupling can be very similar depending on the system. For instance, ultrafast pump-probe transmission recorded for a microcavity strongly coupled to an organic semiconductor in Ref. [33] showed qualitative features identical to the first reported vibrational polariton pump-probe data [36]. The excitonbiexciton ladder described in in Ref. [33] is also notable in the studied context because the energy of the corresponding biexciton is more than twice of the excitonic, which would enable verification of the $\Delta<0$ case of Eq. 25. In fact, whenever electronic transitions are only weakly coupled to high-frequency vibrational modes, and the electronic $S_{1} \rightarrow S_{2}$ (first to second excited-state) transition is dipole-allowed and slightly red- or blue-shifted from the $S_{0} \rightarrow S_{1}$ (ground to first excited-state), we expect electronic TPA rates to have similarly appealing potential for enhancement in optical cavities under the strong coupling regime as discussed in Sec. 3.

It is also notable that, although our model and expressions allow us to derive quantitative nonlinear properties of molecular systems described as three-level system ensembles, the enhancement of molecular nonlinear polarization by intracavity field effects and subnatural polariton linewidths thoroughly discussed in Sec. 2.2 are universal features of the nonlinear susceptibilities of molecular ensembles under strong coupling. In fact, our 
work qualitatively corroborates recently reported nonlinear response enhancement induced by strong coupling of microcavities with organic semiconductor materials $[79,80]$ whose effective Hamiltonian is not given by Eq. 4. Specifically, Barachati and coworkers [79] ascribed third-harmonic generation efficiency gains under cavity strong coupling to intracavity field energy density enhancement, whereas the recent Z-scan measurements reported by Wang et al. [80] showed that polariton resonance effects were also essential to obtain increases in the magnitude of the nonlinear index of refraction and absorption.

In summary, we have derived and analyzed the nonlinear optical susceptibility and TPA rates for a molecular system under strong coupling with an infrared microcavity. By contrasting the polaritonic response with that of bare molecules in free space, we found that enhanced nonlinearities in the strong coupling regime may emerge due to intracavity field enhancement, creation of suitable optical resonances, and subnatural polaritonic linewidths. Our results suggest an increase of several orders of magnitude can potentially be achieved for the polaritonic nonlinear optical response, especially, when a multipolariton transition is resonant with a multiphonon (or multi-electronic state) absorption of the molecular system. Our work suggests new application of molecular polaritonics in two-photon imaging [81], and efficient generation of hot molecular excited-state distributions via (polariton) ladder climbing [82-84], possibly bypassing deleterious intramolecular vibrational relaxation.

Acknowledgments - R.F.R., J.C.G., N.C.G, and J.Y.Z. acknowledge funding support from the Defense Advanced Research Projects Agency under Award No. D19AC00011 for the calculations of nonlinear susceptibilities under strong coupling and the Air Force Office of Scientific Research award FA9550-18-1-0289 for the analysis of optimal conditions for multiphoton absorption under strong light-matter coupling. W.X. thanks the support of NSF CAREER Award DMR1848215.
[1] Shaul Mukamel, Principles of Nonlinear Optical Spectroscopy (Oxford University Press on Demand, 1999).

[2] J. Yuen-Zhou, J.J. Krich, A. Aspuru-Guzik, I. Kassal, and A.S. Johnson, Ultrafast Spectroscopy: Quantum Information and Wavepackets, IOP Expanding Physics (Institute of Physics Publishing, 2014).

[3] Tobias Brixner, Jens Stenger, Harsha M. Vaswani, Minhaeng Cho, Robert E. Blankenship, and Graham R. Fleming, "Two-dimensional spectroscopy of electronic couplings in photosynthesis," Nature 434, 625 (2005).

[4] E. Thyrhaug, R. Tempelaar, M. J. P. Alcocer, K. Žídek, D. Bína, J. Knoester, T. L. C. Jansen, and D. Zigmantas, "Identification and characterization of diverse coherences in the Fenna-Matthews-Olson complex." Nature chemistry 10, 780-786 (2018).

[5] Bo Xiang, Yingmin Li, C. Huy Pham, Francesco Paesani, and Wei Xiong, "Ultrafast direct electron transfer at organic semiconductor and metal interfaces," Science Advances 3, e1701508 (2017).

[6] Ilana Breen, Roel Tempelaar, Laurie A. Bizimana, Benedikt Kloss, David R. Reichman, and Daniel B. Turner, "Triplet Separation Drives Singlet Fission after Femtosecond Correlated Triplet Pair Production in Rubrene," Journal of the American Chemical Society 139, 11745-11751 (2017).

[7] Daniele Sanvitto and Stéphane Kéna-Cohen, "The road towards polaritonic devices," Nature Materials 15, 10611073 (2016).

[8] Anton V. Zasedatelev, Anton V. Baranikov, Darius Urbonas, Fabio Scafirimuto, Ullrich Scherf, Thilo Stöferle, Rainer F. Mahrt, and Pavlos G. Lagoudakis, "A roomtemperature organic polariton transistor," Nature Photonics 13, 378 (2019).

[9] C. Monroe, "Quantum information processing with atoms and photons," Nature 416, 238 (2002).

[10] Samuel L. Braunstein and Peter van Loock, "Quantum information with continuous variables," Reviews of Modern Physics 77, 513-577 (2005).
[11] Dhabih V. Chulhai, Zhongwei Hu, Justin E. Moore, Xing Chen, and Lasse Jensen, "Theory of Linear and Nonlinear Surface-Enhanced Vibrational Spectroscopies," Annual Review of Physical Chemistry 67, 541564 (2016), _eprint: https://doi.org/10.1146/annurevphyschem-040215-112347.

[12] R.W. Boyd and D. Prato, Nonlinear Optics (Elsevier Science, 2008).

[13] Alexey V Kavokin, Jeremy J Baumberg, Guillaume Malpuech, and Fabrice P Laussy, Microcavities, Vol. 21 (Oxford University Press, 2017).

[14] Thomas W. Ebbesen, "Hybrid Light-Matter States in a Molecular and Material Science Perspective," Accounts of Chemical Research 49, 2403-2412 (2016).

[15] Maxim Sukharev and Abraham Nitzan, "Optics of exciton-plasmon nanomaterials," Journal of Physics: Condensed Matter 29, 443003 (2017).

[16] Raphael F. Ribeiro, Luis A. Martínez-Martínez, Matthew Du, Jorge Campos-Gonzalez-Angulo, and Joel Yuen-Zhou, "Polariton chemistry: Controlling molecular dynamics with optical cavities," Chemical Science 9, 6325-6339 (2018).

[17] Maxim Sukharev, Tamar Seideman, Robert J. Gordon, Adi Salomon, and Yehiam Prior, "Ultrafast Energy Transfer between Molecular Assemblies and Surface Plasmons in the Strong Coupling Regime," ACS Nano 8, 807817 (2014).

[18] Parinda Vasa and Christoph Lienau, "Strong Light-Matter Interaction in Quantum Emitter/Metal Hybrid Nanostructures," ACS Photonics 5, 2-23 (2018).

[19] D. S. Dovzhenko, S. V. Ryabchuk, Yu P. Rakovich, and I. R. Nabiev, "Light-matter interaction in the strong coupling regime: Configurations, conditions, and applications," 10, 3589-3605 (2018).

[20] Felipe Herrera and Jeffrey Owrutsky, "Molecular polaritons for controlling chemistry with quantum optics," The Journal of Chemical Physics 152, 100902 (2020).

[21] Vladimir M Agranovich, Excitations in Organic Solids, 
Vol. 142 (OUP Oxford, 2009).

[22] David M. Coles, Niccolo Somaschi, Paolo Michetti, Caspar Clark, Pavlos G. Lagoudakis, Pavlos G. Savvidis, and David G. Lidzey, "Polariton-mediated energy transfer between organic dyes in a strongly coupled optical microcavity," Nature Materials 13, 712 (2014).

[23] Xiaolan Zhong, Thibault Chervy, Lei Zhang, Anoop Thomas, Jino George, Cyriaque Genet, James A. Hutchison, and Thomas W. Ebbesen, "Energy Transfer between Spatially Separated Entangled Molecules," Angewandte Chemie International Edition 56, 9034-9038 (2017).

[24] Matthew Du, Luis A. Martínez-Martínez, Raphael F. Ribeiro, Zixuan Hu, Vinod M. Menon, and Joel Yuen-Zhou, "Theory for polariton-assisted remote energy transfer," Chemical Science 9, 6659-6669 (2018).

[25] E. Orgiu, J. George, J. A. Hutchison, E. Devaux, J. F. Dayen, B. Doudin, F. Stellacci, C. Genet, J. Schachenmayer, C. Genes, G. Pupillo, P. Samorì, and T. W. Ebbesen, "Conductivity in organic semiconductors hybridized with the vacuum field," Nature Materials 14, 1123-1129 (2015).

[26] Johannes Feist and Francisco J. Garcia-Vidal, "Extraordinary Exciton Conductance Induced by Strong Coupling," Physical Review Letters 114, 196402 (2015).

[27] David Hagenmüller, Johannes Schachenmayer, Stefan Schütz, Claudiu Genes, and Guido Pupillo, "CavityEnhanced Transport of Charge," Physical Review Letters 119, 223601 (2017).

[28] Anoop Thomas, Jino George, Atef Shalabney, Marian Dryzhakov, Sreejith J. Varma, Joseph Moran, Thibault Chervy, Xiaolan Zhong, Eloïse Devaux, Cyriaque Genet, James A. Hutchison, and Thomas W. Ebbesen, "Ground-State Chemical Reactivity under Vibrational Coupling to the Vacuum Electromagnetic Field," Angewandte Chemie International Edition 55, 1146211466 (2016).

[29] A. Thomas, L. Lethuillier-Karl, K. Nagarajan, R. M. A. Vergauwe, J. George, T. Chervy, A. Shalabney, E. Devaux, C. Genet, J. Moran, and T. W. Ebbesen, "Tilting a ground-state reactivity landscape by vibrational strong coupling," Science 363, 615-619 (2019).

[30] Jorge A. Campos-Gonzalez-Angulo, Raphael F. Ribeiro, and Joel Yuen-Zhou, "Resonant catalysis of thermally activated chemical reactions with vibrational polaritons," Nature Communications 10, 1-8 (2019).

[31] Tao E. Li, Abraham Nitzan, and Joseph E. Subotnik, "On the Origin of Ground-State Vacuum-Field Catalysis: Equilibrium Consideration," arXiv:2002.09977 [physics] (2020), arXiv:2002.09977 [physics].

[32] Nche T. Fofang, Nathaniel K. Grady, Zhiyuan Fan, Alexander O. Govorov, and Naomi J. Halas, "Plexciton Dynamics: Exciton-Plasmon Coupling in a J-Aggregate$\mathrm{Au}$ Nanoshell Complex Provides a Mechanism for Nonlinearity," Nano Letters 11, 1556-1560 (2011).

[33] T. Virgili, D. Coles, A. M. Adawi, C. Clark, P. Michetti, S. K. Rajendran, D. Brida, D. Polli, G. Cerullo, and D. G. Lidzey, "Ultrafast polariton relaxation dynamics in an organic semiconductor microcavity," Physical Review B 83, 245309 (2011).

[34] Parinda Vasa, Wei Wang, Robert Pomraenke, Melanie Lammers, Margherita Maiuri, Cristian Manzoni, Giulio Cerullo, and Christoph Lienau, "Real-time observation of ultrafast Rabi oscillations between excitons and plas- mons in metal nanostructures with J-aggregates," Nature Photonics 7, 128-132 (2013).

[35] Thibault Chervy, Jialiang Xu, Yulong Duan, Chunliang Wang, Loïc Mager, Maurice Frerejean, Joris A. W. Münninghoff, Paul Tinnemans, James A. Hutchison, Cyriaque Genet, Alan E. Rowan, Theo Rasing, and Thomas W. Ebbesen, "High-Efficiency Second-Harmonic Generation from Hybrid Light-Matter States," Nano Letters 16, 7352-7356 (2016).

[36] A. D. Dunkelberger, B. T. Spann, K. P. Fears, B. S. Simpkins, and J. C. Owrutsky, "Modified relaxation dynamics and coherent energy exchange in coupled vibrationcavity polaritons," Nature Communications 7 (2016), 10.1038/ncomms13504.

[37] Bo Xiang, Raphael F. Ribeiro, Adam D. Dunkelberger, Jiaxi Wang, Yingmin Li, Blake S. Simpkins, Jeffrey C. Owrutsky, Joel Yuen-Zhou, and Wei Xiong, "Twodimensional infrared spectroscopy of vibrational polaritons," Proceedings of the National Academy of Sciences , 201722063 (2018).

[38] Adam D. Dunkelberger, Roderick B. Davidson, Wonmi Ahn, Blake S. Simpkins, and Jeffrey C. Owrutsky, "Ultrafast Transmission Modulation and Recovery via Vibrational Strong Coupling," The Journal of Physical Chemistry A 122, 965-971 (2018).

[39] Aleksandr G. Avramenko and Aaron S. Rury, "Quantum Control of Ultrafast Internal Conversion Using Nanoconfined Virtual Photons," The Journal of Physical Chemistry Letters 11, 1013-1021 (2020).

[40] Courtney A. DelPo, Bryan Kudisch, Kyu Hyung Park, Saeed-Uz-Zaman Khan, Francesca Fassioli, Daniele Fausti, Barry P. Rand, and Gregory D. Scholes, "Polariton Transitions in Femtosecond Transient Absorption Studies of Ultrastrong Light-Molecule Coupling," The Journal of Physical Chemistry Letters 11, 2667-2674 (2020).

[41] Daniel Finkelstein-Shapiro, Pierre-Adrien Mante, Sema Sarisozen, Lukas Wittenbecher, Iulia Minda, Sinan Balci, Tonu Pullerits, and Donatas Zigmantas, "Radiative Transitions and Relaxation Pathways in PlasmonBased Cavity Quantum Electrodynamics Systems," arXiv:2002.05642 [physics, physics:quant-ph] (2020), arXiv:2002.05642 [physics, physics:quant-ph].

[42] Bo Xiang, Raphael F. Ribeiro, Liying Chen, Jiaxi Wang, Matthew Du, Joel Yuen-Zhou, and Wei Xiong, "StateSelective Polariton to Dark State Relaxation Dynamics," The Journal of Physical Chemistry A 123, 5918-5927 (2019).

[43] Raphael F. Ribeiro, Adam D. Dunkelberger, Bo Xiang, Wei Xiong, Blake S. Simpkins, Jeffrey C. Owrutsky, and Joel Yuen-Zhou, "Theory for Nonlinear Spectroscopy of Vibrational Polaritons," The Journal of Physical Chemistry Letters 9, 3766-3771 (2018).

[44] Juan B. Pérez-Sánchez and Joel Yuen-Zhou, "Polariton Assisted Down-Conversion of Photons via Nonadiabatic Molecular Dynamics: A Molecular Dynamical Casimir Effect," The Journal of Physical Chemistry Letters 11, 152-159 (2020).

[45] Federico J. Hernández and Felipe Herrera, "Multi-level quantum Rabi model for anharmonic vibrational polaritons," The Journal of Chemical Physics 151, 144116 (2019).

[46] Daniel A Steck, Classical and Modern Optics, revision 1.7.4 ed. (available online at http://steck.us/teaching, 
2017).

[47] R. Houdré, R. P. Stanley, and M. Ilegems, "Vacuum-field Rabi splitting in the presence of inhomogeneous broadening: Resolution of a homogeneous linewidth in an inhomogeneously broadened system," Physical Review A 53, 2711-2715 (1996).

[48] Jino George, Atef Shalabney, James A. Hutchison, Cyriaque Genet, and Thomas W. Ebbesen, "Liquid-Phase Vibrational Strong Coupling," The Journal of Physical Chemistry Letters 6, 1027-1031 (2015).

[49] J. P. Long and B. S. Simpkins, "Coherent Coupling between a Molecular Vibration and Fabry-Perot Optical Cavity to Give Hybridized States in the Strong Coupling Limit," ACS Photonics 2, 130-136 (2015).

[50] Shaelyn R. Casey and Justin R. Sparks, "Vibrational Strong Coupling of Organometallic Complexes," The Journal of Physical Chemistry C 120, 28138-28143 (2016).

[51] Cristiano Ciuti, Gérald Bastard, and Iacopo Carusotto, "Quantum vacuum properties of the intersubband cavity polariton field," Physical Review B 72, 115303 (2005).

[52] Y. Todorov, A. M. Andrews, R. Colombelli, S. De Liberato, C. Ciuti, P. Klang, G. Strasser, and C. Sirtori, "Ultrastrong Light-Matter Coupling Regime with Polariton Dots," Physical Review Letters 105, 196402 (2010).

[53] Artem Strashko and Jonathan Keeling, "Raman scattering with strongly coupled vibron-polaritons," Physical Review A 94, 023843 (2016).

[54] C. W. Gardiner and M. J. Collett, "Input and output in damped quantum systems: Quantum stochastic differential equations and the master equation," Physical Review A 31, 3761-3774 (1985).

[55] Daniel A Steck, Quantum and Atom Optics, revision 0.12 .0 ed. (available online at http://atomopticsnas.uoregon.edu/ dsteck/teaching/quantumoptics/quantum-optics-notes.pdf, 2017).

[56] Hao Li, Andrei Piryatinski, Jonathan Jerke, Ajay Ram Srimath Kandada, Carlos Silva, and Eric R. Bittner, "Probing dynamical symmetry breaking using quantum-entangled photons," Quantum Science and Technology 3, 015003 (2018).

[57] Marina Litinskaya and Peter Reineker, "Loss of coherence of exciton polaritons in inhomogeneous organic microcavities," Physical Review B 74, 165320 (2006).

[58] Jan Philip Kraack, Angelo Frei, Roger Alberto, and Peter Hamm, "Ultrafast Vibrational Energy Transfer in Catalytic Monolayers at Solid-Liquid Interfaces," The Journal of Physical Chemistry Letters 8, 2489-2495 (2017).

[59] D.P. Craig and T. Thirunamachandran, Molecular Quantum Electrodynamics: An Introduction to RadiationMolecule Interactions, Dover Books on Chemistry Series (Dover Publications, 1998).

[60] Vasil Rokaj, Davis M. Welakuh, Michael Ruggenthaler, and Angel Rubio, "Light-matter interaction in the longwavelength limit: No ground-state without dipole selfenergy," Journal of Physics B: Atomic, Molecular and Optical Physics 51, 034005 (2018).

[61] Simone De Liberato, "Light-Matter Decoupling in the Deep Strong Coupling Regime: The Breakdown of the Purcell Effect," Physical Review Letters 112, 016401 (2014).

[62] Javier del Pino, Johannes Feist, and Francisco J. GarciaVidal, "Quantum theory of collective strong coupling of molecular vibrations with a microcavity mode," New Journal of Physics 17, 053040 (2015).

[63] Zhedong Zhang, Kai Wang, Zhenhuan Yi, M. Suhail Zubairy, Marlan O. Scully, and Shaul Mukamel, "Polariton-Assisted Cooperativity of Molecules in Microcavities Monitored by Two-Dimensional Infrared Spectroscopy," The Journal of Physical Chemistry Letters (2019), 10.1021/acs.jpclett.9b00979.

[64] V. M. Agranovich, M. Litinskaia, and D. G. Lidzey, "Cavity polaritons in microcavities containing disordered organic semiconductors," Physical Review B 67, 085311 (2003).

[65] V. M. Agranovich and Yu. N. Gartstein, "Nature and dynamics of low-energy exciton polaritons in semiconductor microcavities," Physical Review B 75, 075302 (2007).

[66] Thomas Botzung, David Hagenmüller, Stefan Schütz, Jérôme Dubail, Guido Pupillo, and Johannes Schachenmayer, "Dark state localization of quantum emitters in a cavity," arXiv:2003.07179 [cond-mat, physics:quant-ph] (2020), arXiv:2003.07179 [cond-mat, physics:quant-ph].

[67] Crispin W Gardiner and Hermann Haken, Quantum Noise, Vol. 26 (Springer Berlin, 1991).

[68] Jan A. Leegwater and Shaul Mukamel, "Excitonscattering mechanism for enhanced nonlinear response of molecular nanostructures," Physical Review A 46, 452464 (1992).

[69] Ningjun Wang, Vladimir Chernyak, and Shaul Mukamel, "Cooperative ultrafast nonlinear optical response of molecular nanostructures," The Journal of Chemical Physics 100, 2465-2480 (1994).

[70] Vladimir Chernyak, Ningjun Wang, and Shaul Mukamel, "Four-wave mixing and luminescence of confined excitons in molecular aggregates and nanostructures. many-body green function approach," Physics Reports 263, 213-309 (1995).

[71] Merav Muallem, Alexander Palatnik, Gilbert D. Nessim, and Yaakov R. Tischler, "Strong Light-Matter Coupling and Hybridization of Molecular Vibrations in a Low-Loss Infrared Microcavity," The Journal of Physical Chemistry Letters 7, 2002-2008 (2016).

[72] Kishan S. Menghrajani, Geoffrey R. Nash, and William L. Barnes, "Vibrational Strong Coupling with Surface Plasmons and the Presence of Surface Plasmon Stop Bands," ACS Photonics 6, 2110-2116 (2019).

[73] A. Shalabney, J. George, J. Hutchison, G. Pupillo, C. Genet, and T. W. Ebbesen, "Coherent coupling of molecular resonators with a microcavity mode," Nature Communications 6 (2015), 10.1038/ncomms6981.

[74] Max Born and Emil Wolf, Principles of Optics: Electromagnetic Theory of Propagation, Interference and Diffraction of Light (2013).

[75] Michael Tavis and Frederick W. Cummings, "Exact Solution for an N-Molecule-Radiation-Field Hamiltonian," Physical Review 170, 379-384 (1968).

[76] G. V. Varada, M. Sanjay Kumar, and G. S. Agarwal, "Quantum effects of the atom-cavity interaction on four-wave mixing," Optics Communications 62, 328-332 (1987).

[77] Bo Xiang, Raphael F. Ribeiro, Yingmin Li, Adam D. Dunkelberger, Blake B. Simpkins, Joel Yuen-Zhou, and Wei Xiong, "Manipulating optical nonlinearities of molecular polaritons by delocalization," Science Advances 5, eaax5196 (2019).

[78] Chiao-Yu Cheng, Rijul Dhanker, Christopher L. Gray, 
Sukrit Mukhopadhyay, Eric R. Kennehan, John B. Asbury, Anatoliy Sokolov, and Noel C. Giebink, "Charged Polaron Polaritons in an Organic Semiconductor Microcavity," Physical Review Letters 120, 017402 (2018).

[79] Fábio Barachati, Janos Simon, Yulia A. Getmanenko, Stephen Barlow, Seth R. Marder, and Stéphane KénaCohen, "Tunable Third-Harmonic Generation from Polaritons in the Ultrastrong Coupling Regime," ACS Photonics 5, 119-125 (2018).

[80] Kuidong Wang, Marcus Seidel, Kalaivanan Nagarajan, Thibault Chervy, Cyriaque Genet, and Thomas W. Ebbesen, "Large optical nonlinearity enhancement under electronic strong coupling," arXiv:2005.13325 [physics] (2020), arXiv:2005.13325 [physics].

[81] Peter T. C. So, Chen Y. Dong, Barry R. Masters, and Keith M. Berland, "Two-Photon Exci- tation Fluorescence Microscopy," Annual Review of Biomedical Engineering 2, 399-429 (2000), _eprint: https://doi.org/10.1146/annurev.bioeng.2.1.399.

[82] Jan Philip Kraack and Peter Hamm, "Vibrational ladderclimbing in surface-enhanced, ultrafast infrared spectroscopy," Physical Chemistry Chemical Physics 18, 16088-16093 (2016).

[83] Ikki Morichika, Kei Murata, Atsunori Sakurai, Kazuyuki Ishii, and Satoshi Ashihara, "Molecular ground-state dissociation in the condensed phase employing plasmonic field enhancement of chirped mid-infrared pulses," Nature Communications 10, 3893 (2019).

[84] Dominik M. Juraschek, Tomáš Neuman, Johannes Flick, and Prineha Narang, "Cavity control of nonlinear phononics," arXiv:1912.00122 [cond-mat, physics:physics] (2019), arXiv:1912.00122 [cond-mat, physics:physics] 


\title{
Supporting Information for Enhanced optical nonlinearities under strong light-matter coupling
}

\author{
Raphael F. Ribeiro, ${ }^{1}$ Jorge A. Campos-Gonzalez-Angulo, ${ }^{1}$ Noel C. Giebink, ${ }^{2}$ Wei Xiong, ${ }^{1,3}$ and Joel Yuen-Zhou ${ }^{1}$ \\ ${ }^{1}$ Department of Chemistry and Biochemistry, University of California San Diego, La Jolla, CA 92093 \\ ${ }^{2}$ Department of Electrical Engineering, The Pennsylvania State University, University Park, PA, 16802 \\ ${ }^{3}$ Materials Science and Engineering Program, University of California San Diego, La Jolla, CA 92093
}

(Dated: June 16, 2020)

\section{CONTENTS}

1. Basic definitions for empty microcavity[1-3]

2. Nonlinear susceptibility of strongly coupled molecular system

3. Nonlinear absorption spectrum under strong coupling

4. Nonlinear response of bare molecular system

5. Nonlinear absorption spectrum of bare molecular system

6. Quantitative comparison of molecular nonlinear susceptibility of bare and strongly coupled systems

7. Energy eigenvalues and eigenstates of non-dissipative system

References

\section{BASIC DEFINITIONS FOR EMPTY MICROCAVITY[1-3]}

We employ input-output theory $[1,2]$ to describe the open quantum system dynamics of a planar optical microcavity consisting of two highly-reflective symmetric mirrors [4] separated by a distance $L_{c}$. The input radiation is taken to have zero momentum along the transverse direction to the cavity longitudinal axis. Since we work in the limiting case where a single cavity mode interacts with the material system, we only consider the free space electromagnetic modes to the right and left of living in a 1D space with length $L$ sufficiently large for the corresponding field operators to satisfy periodic boundary conditions.

We suppose the system is probed in transmission geometry, where the incident light irradiates the "left" mirror and the optical signal is generated by the photon flux traversing the "right" mirror. The output photon flux is given by

$$
\left\langle\left[b_{\text {out }}^{\mathrm{R}}(t)\right]^{\dagger} b_{\text {out }}(t)\right\rangle,
$$

where the output annihilation operator $b_{\text {out }}(t)$ is written in terms of the "right" free space modes at a future time $t_{1}>t[1]:$

$$
b_{\text {out }}^{\mathrm{R}}(t)=\frac{i}{\sqrt{2 \pi}} \int_{-\infty}^{\infty} \mathrm{d} \omega^{\prime} b_{1}^{\mathrm{R}}\left(\omega^{\prime}\right) e^{-i \omega^{\prime}\left(t-t_{1}\right)}, t_{1}>t
$$

where $b_{1}^{\mathrm{R}}\left(\omega^{\prime}\right)$ is the Heisenberg annihilation operator for a photon with frequency $\omega^{\prime}$ in the free space to the right of the optical cavity at $t_{1}$. In the absence of any input on the system from the right mirror, the input-output relations allow us to directly relate the (right) output EM power at time $t$ with the state of the cavity at the same moment. In particular $[1,2]$,

$$
b_{\text {out }}^{\mathrm{R}}(t)=\sqrt{\frac{\kappa}{2}} b(t)
$$


where $b$ is the cavity mode annihilation operator and $\kappa$ is the total cavity leakage rate (including field decay through both mirrors). The latter is proportional to the mirrors transmission probability $|t|^{2}$, as well as inversely related to the cavity round-trip time $\tau_{c}=2 L_{c} / c,\left(L_{c}\right.$ is the cavity longitudinal length) [3], i.e.,

$$
\kappa=\frac{|t|^{2}}{\tau_{c}}=|t|^{2} \frac{c}{2 L_{c}} .
$$

In this work, we suppose the microcavity is driven by a superposition of coherent state fields which are nearlyresonant and weakly interact with the cavity ( $\kappa$ is much smaller than the cavity photon frequency). The rotating-wave approximation is employed throughout, as is customary in an input-output treatment [2]. We suppose the electric field of the external source which drives the system is expressed as:

$$
\begin{aligned}
E_{\mathrm{in}}^{\mathrm{L}}(t) & =\sum_{\omega>0}\left[E_{\mathrm{in}}^{(+)}(\omega) e^{-i \omega t}+E_{\mathrm{in}}^{(-)}(\omega) e^{i \omega t}\right] \\
& =\sum_{\omega>0} i \sqrt{\frac{\hbar \omega}{2 \epsilon_{0} V}}\left[\alpha_{\mathrm{in}}(\omega) e^{-i \omega t}-\alpha_{\mathrm{in}}^{\dagger}(\omega) e^{i \omega t}\right],
\end{aligned}
$$

where $\epsilon_{0}$ is the free space permittivity, $V=S L$ is the quantization volume of the l.h.s (or r.h.s.) free space, and $\alpha_{\text {in }} \in \mathbb{C}$ is a coherent state amplitude characterizing the phase and intensity of the input external field mode with frequency $\omega$. The photon flux corresponding to each frequency in 5 is given by $\left|\alpha_{\text {in }}(\omega)\right|^{2} c / L^{1}$. The photon input operator is thus ${ }^{2}$

$$
b_{\mathrm{in}}^{\mathrm{L}}(\omega)=i \sqrt{\frac{\mathcal{P}(\omega)}{\hbar \omega}} e^{i \theta_{\mathrm{in}}(\omega)},
$$

where $\mathcal{P}(\omega)=\hbar \omega\left|\alpha_{\text {in }}(\omega)\right|^{2} c / L$ and $\theta_{\text {in }}(\omega)$ is determined by the relationship $\alpha_{\text {in }}(\omega)=\left|\alpha_{\text {in }}(\omega)\right| e^{i \theta_{\text {in }}(\omega)}$

We conclude this section by reviewing some relationships between the cavity electromagnetic field intensity in the presence of steady driving, and the corresponding free space intensity. This identification will be essential for the comparison of the nonlinear optical response of a hybrid cavity with that of the bare molecular material.

First, we recall that the mirrors of a good cavity have nearly vanishing photon transmission probability $|t|^{2} \rightarrow 0$. The cavity is usually characterized by (a) the total photon leakage rate $\kappa$ (Eq. 4) dependent on both geometric parameters (e.g., the cavity length) and the quality of the mirrors (via its dependence on $|t|^{2}$ ), and (b) its finesse coefficient $\mathcal{F}=\pi \sqrt{|r|} /(1-|r|$ ) (where $r$ is the field reflection probability amplitude) [3], which depends only on the quality of the mirrors. As we demonstrate below, the finesse provides a simple measure of the steady-state intracavity (resonant) electromagnetic field intensity $I_{c}$ enhancement compared to free space. In particular, at a cavity antinode, it follows that[3],

$$
I_{c} \approx \frac{2 \mathcal{F}}{\pi} I_{0}
$$

where $I_{0}$ is the free space electromagnetic field intensity [3]. In terms of the finesse, the cavity leakage rate $\kappa$ can be written as

$$
\kappa=\frac{\pi c}{L_{c} \mathcal{F}}
$$

Alternatively, $\mathcal{F}$ is a given as a simple function of the cavity quality factor $Q=\omega_{c} / \kappa[3]$ :

$$
\begin{aligned}
\mathcal{F} & =\frac{\pi c}{L_{c} \omega_{c}} \frac{\omega_{c}}{\kappa} \\
& =\frac{Q}{m}
\end{aligned}
$$

${ }^{1}$ Here, we used the following expression for the mean photon flux

$$
\begin{aligned}
\phi_{\text {in }} & =\frac{S \epsilon_{0} c}{\hbar \omega T} \int_{-T / 2}^{T / 2}\left\langle\left[E_{\mathrm{in}}^{\mathrm{L}}(t)\right]^{\dagger} E_{\mathrm{in}}^{\mathrm{L}}(t)\right\rangle \\
& =\sum_{\omega>0}\left|\alpha_{\mathrm{in}}(\omega)\right|^{2} c / L
\end{aligned}
$$

which is thus given in units of photon number per unit time.

2 Note that our input fields are obtained from superpositions of coherent states of the electromagnetic field in the l.h.s. free space defined by

$$
b_{\mathrm{in}}^{\mathrm{L}}(t)=\frac{i}{\sqrt{2 \pi}} \int_{-\infty}^{\infty} \mathrm{d} \omega^{\prime} b_{0}^{L}\left(\omega^{\prime}\right) e^{-i \omega^{\prime}\left(t-t_{0}\right)}=\sum_{\omega} b_{\mathrm{in}}^{\mathrm{L}}(\omega) e^{-i \omega t},
$$

where $b_{0}^{L}\left(\omega^{\prime}\right)$ is the annihilation operator of the left mode with frequency $\omega^{\prime}$ evaluated at a time $t_{0}<t$. [1] 
where $m \in \mathbb{Z}$ is the longitudinal quantum number of the cavity mode, and we used that the symmetric planar cavity mode frequency corresponding to $m$ is given by $\omega_{c}=c m \pi / L_{c}$.

We conclude this section by deriving the cavity electric field enhancement factor from input-output theory. Consider an empty cavity driven by an external field with power $\mathcal{P}(\omega)=\hbar \omega\left|\alpha_{\mathrm{in}}(\omega)\right|^{2} c / L$, where $\alpha_{\mathrm{in}}(\omega) \in \mathbb{C}$. Using the previously defined parametrization $b_{\mathrm{in}}^{\mathrm{L}}(\omega)=i \sqrt{\mathcal{P}(\omega) / \hbar \omega} e^{i \theta_{\mathrm{in}}(\omega)}$ for the input field, it follows from the input-output treatment of an empty driven cavity that the steady-state positive-frequency component of the empty cavity electric field (in the rotating-wave approximation) $E_{c}^{(+)}(\omega)$ is given by $^{3}$ :

$$
\begin{aligned}
E_{c}^{(+)}(\omega) & =E_{0}^{c} \sqrt{\frac{\kappa}{2}} \frac{-i b_{\mathrm{in}}^{\mathrm{L}}(\omega)}{\omega-\omega_{c}+i \kappa / 2} \\
& =i \sqrt{\frac{\hbar \omega_{c}}{2 \epsilon_{0} S L_{c}}}\left|\alpha_{\mathrm{in}}(\omega)\right| \sqrt{\frac{2 c}{\kappa L}} \frac{\kappa / 2}{\omega-\omega_{c}+i \kappa / 2} e^{i \theta_{\mathrm{in}}(\omega)} \\
& \approx \sqrt{\frac{2 \mathcal{F}}{\pi}} \frac{\kappa / 2}{\omega-\omega_{c}+i \kappa / 2} E_{\mathrm{in}}^{(+)}(\omega) .
\end{aligned}
$$

where we used $E_{0}^{c}=i \sqrt{\hbar \omega_{c} / 2 \epsilon_{0} S L_{c}}$. In the last line we employed $\omega=\omega_{c}+\delta$ and the limit where $\delta \omega / \omega_{c} \rightarrow 0$, i.e., $\omega \approx \omega_{c}$. This approximation is consistent with the weak-coupling and near-resonant assumptions of input-output theory $[1,2]$, and is usually satisfied when $\omega$ is resonant with polaritons in the strong coupling limit (with Rabi splitting significantly weaker than the relevant bare molecule and cavity frequencies).

Equation 14 demonstrates the well-known results that under resonant driving $\left(\omega=\omega_{c}\right)$ (a) the cavity electromagnetic field intensity is enhanced by a factor of $2 \mathcal{F} / \pi$ (at cavity antinodes) compared to free space, and (b) the cavity field is phase-shifted by $-\pi / 2$ relative to the phase of the external field.

\section{NONLINEAR SUSCEPTIBILITY OF STRONGLY COUPLED MOLECULAR SYSTEM}

In this section, we derive the steady-state third-order polarization induced by continuous-wave input fields acting on a molecular system strongly-coupled to an optical cavity as described in the main text. This polarization is the source of the nonlinear optical signal discussed above. The results obtained here are essential for the computation of the nonlinear molecular absorption under strong light-matter coupling which is described in the next section.

To obtain the material nonlinear polarization we solve perturbatively the equations of motion (EOM) for the expectation value of the molecular polarization operator in terms of the driving input fields. We perform this procedure in this subsection assuming the system remains in a pure state at all times.

From the effective Hamiltonian introduced in the main text (Eq. 4), we obtain the Heisenberg-Langevin EOM for the expectation value of the cavity-photon annihilation operator using $i \hbar \partial_{t} b(t)=[b(t), H]-i \hbar \kappa b(t) / 2$ :

$$
\left(i \hbar \frac{\mathrm{d}}{\mathrm{d} t}-\hbar \tilde{\omega}_{c}\right)\langle b(t)\rangle=-i \hbar \sqrt{\frac{\kappa}{2}} b_{\text {in }}^{\mathrm{L}}(t)-\mu \bar{E}_{0}^{c} \sum_{i=1}^{N}\left\langle a_{i}(t)\right\rangle,
$$

where the cavity leakage rate $\kappa$ (derived within input-output theory) was introduced by the replacement $\omega_{c} \rightarrow \tilde{\omega}_{c}=$ $\omega_{c}-i \kappa / 2$. Eq. 15 describes the time-dependent response of the cavity to a collective molecular polarization and to the driving by the input field. Similarly, the molecular response to the cavity electromagnetic field is expressed by the analogous Heisenberg-Langevin EOMs satisfied by the time-dependent single-molecule and collective material

3 This equation can be simply derived by using the Heisenberg equations of motion for the driven cavity mode operator

$$
\begin{aligned}
& \left(i \hbar \partial_{t}-\hbar \tilde{\omega}_{c}\right) b(t)=-i \hbar \sqrt{\frac{\kappa}{2}} b_{\text {in }}^{\mathrm{L}}(t) \\
& \Longrightarrow b(\omega)=-i \hbar \sqrt{\frac{\kappa}{2}} \frac{b_{\text {in }}^{\mathrm{L}}(\omega)}{\hbar \omega-\hbar \omega_{c}+i \kappa / 2}, \\
& \Longrightarrow E_{c}^{(+)}(\omega)=i \sqrt{\frac{\hbar \omega_{c}}{2 \epsilon_{0} S L_{c}}} b(\omega)=\sqrt{\frac{\hbar \omega_{c}}{2 \epsilon_{0} S L_{c}}} \sqrt{\frac{\kappa}{2}} \frac{b_{\text {in }}^{\mathrm{L}}(\omega)}{\omega-\omega_{c}+i \kappa / 2},
\end{aligned}
$$

where we used $\tilde{\omega}_{c}=\omega_{c}-i \kappa / 2$, and $b(t)=\sum_{\omega} b(\omega) e^{-i \omega t}$. 
polarizations:

$$
\begin{aligned}
\left(i \hbar \frac{\mathrm{d}}{\mathrm{d} t}-\hbar \tilde{\omega}_{0}\right)\left\langle\mu a_{i}(t)\right\rangle & =-\mu^{2} E_{0}^{c}\langle b(t)\rangle-2 \hbar \Delta \mu\left\langle a_{i}^{\dagger}(t) a_{i}(t) a_{i}(t)\right\rangle \\
& =-\mu^{2} E_{0}^{c}\langle b(t)\rangle-2 \hbar \Delta \mu\left\langle a_{i}^{\dagger}(t)\right\rangle\left\langle a_{i}(t) a_{i}(t)\right\rangle, \\
\left(i \hbar \frac{\mathrm{d}}{\mathrm{d} t}-\hbar \tilde{\omega}_{0}\right)\left\langle\mu \sum_{i=1}^{N} a_{i}(t)\right\rangle & =-N \mu^{2} E_{0}^{c}\langle b(t)\rangle-2 \hbar \Delta \mu\left\langle\sum_{i=1}^{N} a_{i}^{\dagger}(t) a_{i}(t) a_{i}(t)\right\rangle \\
& =-N \mu^{2} E_{0}^{c}\langle b(t)\rangle-2 \hbar \Delta \mu \sum_{i=1}^{N}\left\langle a_{i}^{\dagger}(t)\right\rangle\left\langle a_{i}(t) a_{i}(t)\right\rangle,
\end{aligned}
$$

where $\tilde{\omega}_{0}=\omega_{0}-i \gamma_{m} / 2$, and we obtained the final equations in each case using the factorization property of normal-ordered (all annihilation operators are to the right of the creation) pure-state correlation functions which, for $\left\langle a^{\dagger}(t) a(t) a(t)\right\rangle$ is valid to $O\left(\left|E_{\text {in }}\right|^{3}\right.$ ) (see e.g., Ref. [5]). Hence, Eqs. 16 and 17 are valid to $O\left(\left|E_{\text {in }}\right|^{3}\right){ }^{4}$.

Each of the time-dependent expectation values appearing in the Eqs. 15 and 17 admits an expansion in powers of the input field $\left\langle b_{\mathrm{in}}^{\mathrm{L}}\right\rangle$ (since the cavity is only weakly-coupled to the external modes). For instance, we can write $\left\langle a_{i}(t)\right\rangle=$ $\sum_{p}\left\langle a_{i}(t)\right\rangle^{(p)}$, where $\left\langle a_{i}(t)\right\rangle^{p}=\mathrm{O}\left[\left(b_{\mathrm{in}}^{\mathrm{L}}\right)^{p}\right]$. Hereafter, we will employ the following frequency-domain expansion of the expectation value of time-dependent operators, e.g.,

$$
\langle O\rangle(t)=\sum_{\omega}\langle O\rangle(\omega) e^{-i \omega t}=\sum_{\omega>0}\left(\langle O\rangle^{(+)}(\omega) e^{-i \omega t}+\langle O\rangle^{(-)}(\omega) e^{i \omega t}\right) .
$$

Performing an expansion of both sides of Eqs. 15 and 17 in powers of the input electric field amplitude we find the third-order contribution to the cavity and molecular annihilation operator expectation values satisfy the following coupled equations in the frequency domain:

$$
\begin{aligned}
& \left(\hbar \omega-\hbar \tilde{\omega}_{c}\right)\langle b\rangle^{(3)}(\omega)=-\mu \bar{E}_{0}^{c} \sum_{i=1}^{N}\left\langle a_{i}\right\rangle^{(3)}(\omega), \\
& \left(\hbar \omega-\hbar \tilde{\omega}_{0}\right) \sum_{i=1}^{N}\left\langle\mu a_{i}\right\rangle^{(3)}(\omega)=-N \mu^{2} E_{0}^{c}\langle b\rangle^{(3)}(\omega)-2 \hbar \Delta \mu \sum_{\omega_{a}} \sum_{\omega_{b}} \sum_{i=1}^{N}\left\langle a_{i}^{\dagger}\right\rangle^{(1)}\left(-\omega_{a}\right)\left\langle a_{i} a_{i}\right\rangle^{(2)}\left(\omega_{b}\right) \delta_{\omega,-\omega_{a}+\omega_{b} .}
\end{aligned}
$$

The positive frequency material third-order polarization component with frequency $\omega$ is given by $\langle P\rangle^{(3)}(\omega)=$ $\mu \sum_{i=1}^{N}\left\langle a_{i}\right\rangle^{(3)}(\omega)$. As shown above, it can be expressed in terms of the photonic variable $\langle b\rangle^{(3)}(\omega)$ and lower-order molecular correlators. Inserting the formal solution of Eq. 20 into Eq.19, we find:

$$
\begin{aligned}
& \langle b\rangle^{(3)}(\omega)=2 \hbar \Delta \mu \bar{E}_{0}^{c} \sum_{\omega_{a} \omega_{b}} \sum_{i=1}^{N} \frac{\left\langle a_{i}^{\dagger}\right\rangle^{(1)}\left(-\omega_{a}\right)\left\langle a_{i} a_{i}\right\rangle^{(2)}\left(\omega_{b}\right)}{\left(\hbar \omega-\hbar \tilde{\omega}_{c}\right)\left(\hbar \omega-\hbar \tilde{\omega}_{0}\right)-N\left|\mu E_{0}^{c}\right|^{2}} \delta_{\omega,-\omega_{a}+\omega_{b},} \\
& \sum_{i=1}^{4} \mu\left\langle a_{i}\right\rangle^{(3)}(\omega)=-2 \hbar \Delta \mu \sum_{\omega_{a} \omega_{b}} \sum_{i=1}^{N} \frac{\hbar \omega-\hbar \tilde{\omega}_{c}}{\left(\hbar \omega-\hbar \tilde{\omega}_{c}\right)\left(\hbar \omega-\hbar \tilde{\omega}_{0}\right)-N\left|\mu E_{0}^{c}\right|^{2}}\left\langle a_{i}^{\dagger}\right\rangle^{(1)}\left(-\omega_{a}\right)\left\langle a_{i} a_{i}\right\rangle^{(2)}\left(\omega_{b}\right) \delta_{\omega,-\omega_{a}+\omega_{b}} .
\end{aligned}
$$

4 More explicitly, we used,

$$
\begin{aligned}
\left\langle a_{i}^{\dagger}(t) a_{i}(t) a_{i}(t)\right\rangle & =\left\langle\psi\left|a_{i}^{\dagger}(t) a_{i}(t) a_{i}(t)\right| \psi\right\rangle \\
& =\left\langle\psi(t)\left|a_{i}^{\dagger} a_{i} a_{i}\right| \psi(t)\right\rangle \\
& =\sum_{n_{i}}\left\langle\psi(t)\left|a_{i}^{\dagger}\right| n_{i}\right\rangle\left\langle n_{i}\left|a_{i} a_{i}\right| \psi(t)\right\rangle \\
& =\sum_{n_{i}} c_{n_{i}+1}^{*}(t) \sqrt{\left(n_{i}+1\right)\left(n_{i}+1\right)\left(n_{i}+2\right)} c_{n+2}(t) \\
& =\underbrace{c_{1}^{*}(t) \sqrt{2} c_{2}(t)}_{=O\left(\left|E_{\mathrm{in}}\right|^{3}\right)}+O\left(\left|E_{\mathrm{in}}\right|^{5}\right) \\
& =\left\langle\psi(t)\left|a_{i}^{\dagger}\right| 0\right\rangle\left\langle 0\left|a_{i} a_{i}\right| \psi(t)\right\rangle+O\left(\left|E_{\mathrm{in}}\right|^{5}\right) \\
& \approx \underbrace{\left\langle\psi(t)\left|a_{i}^{\dagger}\right| \psi(t)\right\rangle}_{=O\left(\left|E_{\mathrm{in}}\right|\right)} \underbrace{\left\langle\psi(t)\left|a_{i} a_{i}\right| \psi(t)\right\rangle}_{=O\left(\left|E_{\mathrm{in}}\right|^{2}\right)} \\
& \approx\left\langle a_{i}^{\dagger}(t)\right\rangle\left\langle a_{i}(t) a_{i}(t)\right\rangle,
\end{aligned}
$$

where we used the resolution of the identity for the $i$ th oscillator $I_{i}=\sum\left|n_{i}\right\rangle\left\langle n_{i}\right|$ in terms of Fock states $\left\{\left|n_{i}\right\rangle\right\}$, and kept contributions to the wavefunction $|\psi(t)\rangle$ to $O\left(\left|E_{\text {in }}\right|^{3}\right)$. 
The first-order molecular expectation values $\left\langle\mu a_{i}\left(\omega_{a}\right)\right\rangle^{(1)}$ describe the linear polarization induced on each molecule. By solving the coupled cavity-matter equations (Eqs. 15 and 17) to first-order in the input field, we can obtain the linear molecular polarization in the strongly coupled device. In the frequency domain the equations to be solved are:

$$
\begin{aligned}
& \left(\hbar \omega-\hbar \tilde{\omega}_{c}\right)\langle b\rangle^{(1)}(\omega)=-i \hbar \sqrt{\frac{\kappa}{2}} b_{\mathrm{in}}^{\mathrm{L}}(\omega)-\mu \bar{E}_{0}^{c} \sum_{i=1}^{N}\left\langle a_{i}\right\rangle^{(1)}(\omega), \\
& \left(\hbar \omega-\hbar \tilde{\omega}_{0}\right) \sum_{i=1}^{N}\left\langle a_{i}\right\rangle^{(1)}(\omega)=-N \mu E_{0}^{c}\langle b\rangle^{(1)}(\omega) .
\end{aligned}
$$

The explicit solution for the linear polarization $\langle P\rangle^{(1)}(\omega) \equiv \sum_{i=1}^{N}\left\langle\mu a_{i}\right\rangle^{(1)}(\omega)$ induced by the input field is given by:

$$
\begin{aligned}
\langle P\rangle^{(1)}(\omega) & =i \hbar \sqrt{\frac{\kappa}{2}} \frac{N \mu^{2} E_{0}^{c} b_{\text {in }}^{\mathrm{L}}(\omega)}{\left(\hbar \omega-\hbar \tilde{\omega}_{c}\right)\left(\hbar \omega-\hbar \tilde{\omega}_{0}\right)-\left|\mu E_{0}^{c}\right|^{2} N} \\
& =N \mu G_{m m}(\omega) \mu\left[E_{0}^{c} G_{p p}^{(0)}(\omega) i \hbar \sqrt{\frac{\kappa}{2}} b_{\text {in }}^{\mathrm{L}}(\omega)\right]
\end{aligned}
$$

where $G_{p p}^{(0)}(\omega)=1 /\left(\hbar \omega-\hbar \tilde{\omega}_{c}\right)$ is the bare cavity photon frequency-domain propagator, and $G_{m m}(\omega)$ is the singlemolecule response function renormalized due to the material strong interaction with the optical cavity

$$
G_{m m}(\omega)=\frac{1}{\hbar \omega-\hbar \tilde{\omega}_{0}-\frac{\left|\mu E_{0}^{c}\right|^{2} N}{\hbar \omega-\hbar \tilde{\omega}_{c}}}
$$

Note the light-matter weak-coupling limit for the molecular response function $G_{m m}^{(0)}=1 /\left(h b a r \omega-\hbar \tilde{\omega}_{0}\right)$ can be straightforwardly obtained from the above expression by performing a power series expansion in terms of $\left|\mu E_{0}^{c}\right|$. The linear response induced by the external field on the cavity photon is similarly given by:

$$
\begin{aligned}
\langle b\rangle^{(1)}(\omega) & =-i \hbar \sqrt{\frac{\kappa}{2}} \frac{\left(\hbar \omega-\hbar \tilde{\omega}_{0}\right) b_{\text {in }}^{\mathrm{L}}(\omega)}{\left(\hbar \omega-\hbar \tilde{\omega}_{c}\right)\left(\hbar \omega-\hbar \tilde{\omega}_{0}\right)-\left|\mu E_{0}^{c}\right|^{2} N} \\
& =G_{p p}(\omega)\left[-i \hbar \sqrt{\frac{\kappa}{2}} b_{\text {in }}^{\mathrm{L}}(\omega)\right]
\end{aligned}
$$

where $G_{p p}(\omega)$ is the frequency-domain representation of the cavity photon retarded propagator under strong coupling conditions

$$
G_{p p}(\omega)=\frac{1}{\hbar \omega-\hbar \tilde{\omega}_{c}-\frac{\left|\mu E_{0}^{c}\right|^{2} N}{\hbar \omega-\hbar \tilde{\omega}_{0}}}
$$

Note that the hybrid cavity linear response field amplitude given by Eq. 27 has the same form as that for an empty cavity (12). The bare cavity result is obtained trivially by simply taking $\mu \rightarrow 0$ in Eq. 27. The following relationship between the cavity and molecular polarization retarded Green functions will be useful later:

$$
G_{p p}(\omega)=G_{p p}^{(0)}(\omega) \frac{G_{m m}(\omega)}{G_{m m}^{(0)}(\omega)}
$$

The last expectation value which we need to compute in order to obtain the hybrid cavity third-order response is $\left\langle a_{i}(t) a_{i}(t)\right\rangle^{(2)}$ (see Eq. 21). The time-dependence of this function is coupled to the other totally-symmetric (with respect to permutation of the molecular indices) 2-particle variables of the system, namely, $\langle b(t) b(t)\rangle^{(2)}$ which describes the evolution of the two-cavity photon state, $\left\langle a_{i}(t) b(t)\right\rangle^{(2)}$ which probes the correlated propagation of a photon and the $i$ th molecule phonon, and $\left\langle a_{i}(t) a_{j}(t)\right\rangle^{(2)}, i \neq j$, that describes propagation of vibrational excited-states in distinct molecules.

The system of Heisenberg-Langevin equations for the bright two-particle variables mentioned above can be derived using the operator equations of motion generated Hamiltonian in Eq. (4) of the main text, together with the same replacements effected above $\omega_{0} \rightarrow \omega_{0}-i \gamma_{m} / 2$, and $\omega_{c} \rightarrow \omega_{c}-i \kappa / 2$. It follows from the input-output treatment [2] 
that under the assumptions of Markovian molecular bath, and in the absence of an input molecular polarization, the resulting two-particle EOMs are given by:

$$
\begin{aligned}
& {\left[i \hbar \frac{\mathrm{d}}{\mathrm{d} t}-2\left(\hbar \tilde{\omega}_{0}-\hbar \Delta\right)\right]\left\langle a_{i}(t) a_{i}(t)\right\rangle^{(2)}=-2 \mu E_{0}^{c}\left\langle a_{i}(t) b(t)\right\rangle^{(2)},} \\
& {\left[i \hbar \frac{\mathrm{d}}{\mathrm{d} t}-\left(\hbar \tilde{\omega}_{c}+\hbar \tilde{\omega}_{0}\right)\right]\left\langle a_{i}(t) b(t)\right\rangle^{(2)}=-\mu E_{0}^{c}\langle b(t) b(t)\rangle^{(2)}-\mu \bar{E}_{0}^{c} \sum_{j=1}^{N}\left\langle a_{i}(t) a_{j}(t)\right\rangle^{(2)}-i \hbar \sqrt{\frac{\kappa}{2}} b_{\mathrm{in}}^{\mathrm{L}}(t)\left\langle a_{i}(t)\right\rangle^{(1)},} \\
& {\left[i \hbar \frac{\mathrm{d}}{\mathrm{d} t}-2 \hbar \tilde{\omega}_{c}\right]\langle b(t) b(t)\rangle^{(2)}=-\mu \bar{E}_{0}^{c} \sum_{i}\left\langle a_{i}(t) b(t)\right\rangle^{(2)}-2 i \hbar \sqrt{\frac{\kappa}{2}} b_{\text {in }}(t)\langle b(t)\rangle^{(1)},} \\
& {\left[i \hbar \frac{\mathrm{d}}{\mathrm{d} t}-2 \hbar \tilde{\omega}_{0}\right]\left\langle a_{i}(t) a_{j}(t)\right\rangle^{(2)}=-\mu E_{0}^{c}\left[\left\langle a_{i}(t) b(t)\right\rangle^{(2)}+\left\langle a_{j}(t) b(t)\right\rangle^{(2)}\right], \quad j \neq i .}
\end{aligned}
$$

These equations show, as expected, that two-particle states are driven by the input field only in the presence of nonvanishing first-order photonic or molecular polarization (represented by $\langle b(t)\rangle^{(1)}$ and $\left\langle a_{i}(t)\right\rangle^{(1)}$ ). To solve this system in the frequency domain, we note that the electromagnetic field interacts equally with each molecule, and therefore, $\left\langle a_{i}(t) b(t)\right\rangle=\left\langle a_{j}(t) b(t)\right\rangle$, for all $i, j \in\{1, \ldots, N\}$. From the same argument, it also follows that the correlators $\left\langle a_{i}(t) a_{j}(t)\right\rangle_{i \neq j}$, and $\left\langle a_{i}(t) a_{i}(t)\right\rangle$ are independent of the molecular indices. These considerations imply that, while the system of two-particle eqs. given above has $(N+1)^{2}$ unknowns, only four of those are independent. In order to proceed, we need $\left\langle a_{i} a_{i}\right\rangle^{(2)}(\omega)$ which can be written as:

$$
\left\langle a_{i} a_{i}\right\rangle^{(2)}(\omega)=\frac{2\left(\hbar \omega-2 \hbar \tilde{\omega}_{0}\right)}{D(\omega)}\left(\mu E_{0}^{c}\right)^{2} f_{\mathrm{ext}}^{b b}(\omega)-\frac{2\left(\hbar \omega-2 \hbar \tilde{\omega}_{c}\right)\left(\hbar \omega-2 \hbar \tilde{\omega}_{0}\right)}{D(\omega)} \mu E_{0}^{c} f_{\mathrm{ext}}^{m b}(\omega),
$$

where $f_{\text {ext }}^{b b}(\omega)=-2 i \hbar \sqrt{\frac{\kappa}{2}}\left\langle b_{\mathrm{in}}^{\mathrm{L}} b^{(1)}\right\rangle^{(2)}(\omega)$ and $f_{\text {ext }}^{m b}(\omega)=-i \hbar \sqrt{\frac{\kappa}{2}}\left\langle b_{\text {in }}^{\mathrm{L}} a_{i}^{(1)}\right\rangle^{(2)}(\omega)$, and $D(\omega)$ is a 4th-order polynomial, with its roots corresponding to the bright resonances of the doubly-excited manifold of the system. Denoting by $D^{(0)}(\omega)$ the bare noninteracting 2-particle resonances

$$
D^{(0)}(\omega)=\left(\hbar \omega-\hbar \tilde{\omega}_{c}-\hbar \tilde{\omega}_{0}\right)\left(\hbar \omega-2 \hbar \tilde{\omega}_{0}+2 \hbar \Delta\right)\left(\hbar \omega-2 \hbar \tilde{\omega}_{c}\right)\left(\hbar \omega-2 \hbar \tilde{\omega}_{0}\right)
$$

it follows that the interacting complex two-particle energy eigenvalues are given by the roots of

$$
\begin{aligned}
D(\omega)= & D^{(0)}(\omega)-2 g^{2} N\left(\hbar \omega-2 \hbar \tilde{\omega}_{0}\right)\left(\hbar \omega-2 \hbar \tilde{\omega}_{0}+2 \hbar \Delta\right)-2 g^{2}(N-1)\left(\hbar \omega-2 \hbar \tilde{\omega}_{0}+2 \hbar \Delta\right)\left(\hbar \omega-2 \hbar \tilde{\omega}_{c}\right) \\
& -2 g^{2}\left(\hbar \omega-2 \hbar \tilde{\omega}_{c}\right)\left(\hbar \omega-2 \hbar \tilde{\omega}_{0}\right),
\end{aligned}
$$

where $g^{2}=\left|\mu E_{0}^{c}\right|^{2}$ as in the main text. We can also write Eq. 34 in terms of retarded single and two-particle Green functions in the frequency domain:

$$
\begin{aligned}
\left\langle a_{i} a_{i}\right\rangle^{(2)}(\omega) & =-2 i \hbar \sqrt{\frac{\kappa}{2}} G_{m m, p p}(\omega)\left(\mu E_{0}^{c}\right)^{2}\left\langle b_{\mathrm{in}}^{\mathrm{L}} b^{(1)}\right\rangle(\omega)+i \hbar \sqrt{\frac{\kappa}{2}} G_{m m, m p}(\omega) \mu E_{0}^{c}\left\langle b_{\mathrm{in}}^{\mathrm{L}} a_{i}^{(1)}\right\rangle(\omega), \\
& =-\hbar^{2} \frac{\kappa}{2} \sum_{u v}\left[2 G_{m m, p p}\left(\omega_{u}+\omega_{v}\right) G_{p p}\left(\omega_{u}\right)+G_{m m, m p}\left(\omega_{u}+\omega_{v}\right) G_{m m}\left(\omega_{u}\right) G_{p p}^{(0)}\left(\omega_{u}\right)\right]\left(\mu E_{0}^{c}\right)^{2} b_{\mathrm{in}}^{\mathrm{L}}\left(\omega_{v}\right) b_{\mathrm{in}}^{\mathrm{L}}\left(\omega_{u}\right) \delta_{\omega, \omega_{u}+\omega_{v}}
\end{aligned}
$$

where $G_{m m, p p}(\omega)$ corresponds to the Fourier transform of the probability amplitude for a two-cavity photon state to undergo a transition into a state where a given molecule is doubly excited, and $G_{m m, m p}(\omega)$ is the transition amplitude into the doubly-excited state of a given molecule from an initial state containing a photon and a single vibrational excitation of the same molecule. These propagators can be written explicitly as:

$$
\begin{aligned}
G_{m m, p p}(\omega) & =\frac{2\left(\hbar \omega-2 \hbar \omega_{0}+i \hbar \gamma_{m}\right)}{D(\omega)}, \\
G_{m m, m p}(\omega) & =\frac{2\left(\hbar \omega-2 \hbar \omega_{c}+i \hbar \kappa\right)\left(\hbar \omega-2 \hbar \omega_{0}+i \hbar \gamma_{m}\right)}{D(\omega)} .
\end{aligned}
$$

Using the relation introduced in Eq. 29, we rewrite the two-particle molecular response as:

$$
\begin{aligned}
\left\langle a_{i} a_{i}\right\rangle^{(2)}(\omega)= & \frac{1}{2} \sum_{u v}\left\{2 G_{m m, p p}\left(\omega_{u}+\omega_{v}\right)\left[G_{m m}^{(0)}\left(\omega_{u}\right)\right]^{-1}\left[G_{p p}^{(0)}\left(\omega_{v}\right)\right]^{-1}+G_{m m, m p}\left(\omega_{u}+\omega_{v}\right)\left[G_{p p}^{(0)}\left(\omega_{v}\right)\right]^{-1}\right\} \\
& \times G_{m m}\left(\omega_{u}\right)\left(\mu E_{0}^{c}\right)^{2}\left[-i \hbar \sqrt{\frac{\kappa}{2}} G_{p p}^{(0)}\left(\omega_{v}\right) b_{\mathrm{in}}^{\mathrm{L}}\left(\omega_{v}\right)\right]\left[-i \hbar \sqrt{\frac{\kappa}{2}} G_{p p}^{(0)}\left(\omega_{u}\right) b_{\mathrm{in}}^{\mathrm{L}}\left(\omega_{u}\right)\right] \delta_{\omega, \omega_{u}+\omega_{v}} .
\end{aligned}
$$


By symmetrizing the summand of the previous equation, we obtain:

$$
\begin{aligned}
\left\langle a_{i} a_{i}\right\rangle^{(2)}(\omega)= & \sum_{u v} \Gamma_{m m, m m}\left(\omega_{u}+\omega_{v}\right) G_{m m}\left(\omega_{u}\right) G_{m m}\left(\omega_{v}\right)\left[-\mu E_{0}^{c} i \hbar \sqrt{\frac{\kappa}{2}} G_{p p}^{(0)}\left(\omega_{v}\right) b_{\mathrm{in}}^{\mathrm{L}}\left(\omega_{v}\right)\right]\left[-\mu E_{0}^{c} i \hbar \sqrt{\frac{\kappa}{2}} G_{p p}^{(0)}\left(\omega_{u}\right) b_{\mathrm{in}}^{\mathrm{L}}\left(\omega_{u}\right)\right] \\
& \times \delta_{\omega, \omega_{u}+\omega_{v}},
\end{aligned}
$$

where $\Gamma$ is the two-particle scattering matrix, and $\Gamma_{m m, m m}$ is the amplitude for the elastic scattering of two excitations in the same molecule. It may be written as:

$$
\Gamma_{m m, m m}(\omega)=\frac{\left(\hbar \omega-2 \hbar \tilde{\omega}_{0}\right)\left(\hbar \omega-\hbar \tilde{\omega}_{0}-\hbar \tilde{\omega}_{c}\right)\left[\left(\hbar \omega-2 \hbar \tilde{\omega}_{0}\right)\left(\hbar \omega-2 \hbar \tilde{\omega}_{c}\right)-4 g^{2} N\right]}{D\left(\omega_{u}+\omega_{v}\right)} .
$$

The nonlinear component of the molecular polarization $\left\langle P\left(\omega_{s}\right)\right\rangle^{(3)}=\mu \sum_{i=1}^{N}\left\langle a_{i}\left(\omega_{s}\right)\right\rangle^{(3)}$ can now be given the explicit form:

$$
\begin{aligned}
\left\langle P\left(\omega_{s}\right)\right\rangle^{(3)}= & \sum_{\omega_{u} \omega_{v} \omega_{w}} 2 N \hbar \Delta \mu^{4} G_{m m}\left(\omega_{s}\right) \bar{G}_{m m}\left(\omega_{w}\right) \Gamma_{m m, m m}\left(\omega_{u}+\omega_{v}\right) G_{m m}\left(\omega_{v}\right) G_{m m}\left(\omega_{u}\right) \times \\
& G_{p p}^{(0)}\left(\omega_{v}\right) \bar{G}_{p p}^{(0)}\left(\omega_{w}\right) G_{p p}^{(0)}\left(\omega_{u}\right)\left(\frac{\hbar \kappa}{2} \sqrt{\frac{2 \mathcal{F}}{\pi}}\right)^{3} E_{\mathrm{in}}^{(+)}\left(\omega_{v}\right) E_{\mathrm{in}}^{(-)}\left(\omega_{w}\right) E_{\mathrm{in}}^{(+)}\left(\omega_{u}\right) \delta_{\omega_{s}, \omega_{v}-\omega_{w}+\omega_{u}}
\end{aligned}
$$

where we used $-i E_{0}^{c} \sqrt{\frac{\kappa}{2}} b_{\text {in }}^{\mathrm{L}}(\omega) \approx \frac{\kappa}{2} \sqrt{\frac{2 \mathcal{F}}{\pi}} E_{\text {in }}^{(+)}(\omega)$ (from Eq. 14). From the above expression and the definition of the molecular nonlinear susceptibility [6], we find

$\chi^{(3)}\left(-\omega_{s} ; \omega_{v},-\omega_{w}, \omega_{u}\right)=2 \hbar \Delta N \mu G_{m m}\left(\omega_{s}\right) \bar{G}_{m m}\left(\omega_{w}\right) \Gamma_{m m, m m}\left(\omega_{u}+\omega_{v}\right) G_{m m}\left(\omega_{u}\right) G_{m m}\left(\omega_{v}\right) \times$

$$
\left[\mu \sqrt{\frac{2 \mathcal{F}}{\pi}} \frac{\hbar \kappa}{2} G_{p p}^{(0)}\left(\omega_{v}\right)\right]\left[\mu \sqrt{\frac{2 \mathcal{F}}{\pi}} \frac{\hbar \kappa}{2} \bar{G}_{p}^{(}\right.
$$

\section{NONLINEAR ABSORPTION SPECTRUM UNDER STRONG COUPLING}

In this section, we compute the nonlinear part of the absorption spectrum of an optical microcavity strongly coupled to the molecular polarization. In particular, we will calculate the nonlinear part (in the input electric field amplitude) of the external field power dissipated by the molecular system under steady-state conditions.

Mathematically, the steady-state regime is characterized by a time-independent molecular excited-state population i.e., $\partial_{t} \sum_{i=1}^{N}\left[a_{i}^{\dagger}(t) a_{i}(t)\right]=0$. Using the Heisenberg-Langevin equation for $\sum_{i=1}^{N} a_{i}^{\dagger}(t) a_{i}(t)$, we find that steady-state implies:

$$
\begin{aligned}
0 & =-i \hbar \gamma_{m} \sum_{i=1}^{N} a_{i}^{\dagger}(t) a_{i}(t)+\mu\left[\bar{E}_{0}^{c} b^{\dagger}(t) \sum_{i=1}^{N} a_{i}(t)-E_{0}^{c} \sum_{i=1}^{N} a_{i}^{\dagger}(t) b(t)\right] \\
& \Longrightarrow \sum_{i=1}^{N} \gamma_{m} a_{i}^{\dagger} a_{i}=2 \operatorname{Im}\left[\sum_{i=1}^{N} \frac{\mu \bar{E}_{0}^{c}}{\hbar} b^{\dagger} a_{i}\right]
\end{aligned}
$$

The last equality expresses the balance between the steady-state rate of molecular excited-state decay (l.h.s.) and driving by the external field mediated by the cavity (r.h.s). Hence, the photon absorption rate by the molecular system can be written as:

$$
W=\frac{2}{\hbar} \operatorname{Im}\left\langle E_{c}^{\dagger} P\right\rangle_{\mathrm{ss}},
$$

where $E_{c}^{\dagger}=\bar{E}_{0}^{c} b^{\dagger}$ and $P$ are the (complex conjugate) cavity electric field amplitude and collective molecular polarization in steady-state, respectively. Both $E_{c}$ and $P$ admit power series expansions in the external fields (see Sec. 2). The first non-vanishing nonlinear terms scales cubically with the input field $b_{\mathrm{in}}^{\mathrm{L}}$. Therefore, it follows that the nonlinear response contribution to the photon absorption rate $W$ scales as $\left|E_{\text {in }}\right|^{4}$. To obtain this quantity, we will solve the 
coupled Heisenberg-Langevin EOMs for population and coherence variables in the presence of driving by the external input fields. From now on, we will denote steady-state quantities by the usual expectation value notation without the subscript "ss", as we will always work under steady-state conditions. Moreover, we will disregard the frequency dependence of all quantities until we obtain the final expression for the nonlinear absorption. In this section, we take the input field to be a monochromatic beam, i.e., $b_{i n}\left(\omega^{\prime}\right)=0$ for all $\omega^{\prime} \neq \omega$.

In steady-state, the cavity-molecular polarization coherence $\left\langle E_{c}^{\dagger} P\right\rangle$ satisfies

$$
\left(\hbar \tilde{\omega}_{c}^{*}-\hbar \tilde{\omega}_{0}\right)\left\langle E_{c}^{\dagger} P\right\rangle=-\mu^{2}\left|E_{0}^{c}\right|^{2}\left(N\left\langle b^{\dagger} b\right\rangle-\sum_{i j=1}^{N}\left\langle a_{i}^{\dagger} a_{j}\right\rangle\right)-2 \hbar \Delta \mu \sum_{i=1}^{N}\left\langle E_{c}^{\dagger} a_{i}^{\dagger} a_{i} a_{i}\right\rangle-i \hbar \sqrt{\frac{\kappa}{2}}\left\langle\bar{E}_{0}^{c}\left(b_{\mathrm{in}}^{\mathrm{L}}\right)^{\dagger} P\right\rangle .
$$

Because we only care about the $O\left(\left|b_{\mathrm{in}}^{\mathrm{L}}\right|^{4}\right)$ absorption component, and we have assumed our system is in a pure-state, it follows by the same argument employed in Sec. 2 that $\left\langle b^{\dagger} a_{i}^{\dagger} a_{i} a_{i}\right\rangle=\left\langle b^{\dagger} a_{i}^{\dagger}\right\rangle\left\langle a_{i} a_{i}\right\rangle$. Thus,

$$
\left(\hbar \tilde{\omega}_{c}^{*}-\hbar \tilde{\omega}_{0}\right)\left\langle E_{c}^{\dagger} P\right\rangle^{(4)}=-\mu^{2}\left|E_{0}^{c}\right|^{2}\left(N\left\langle b^{\dagger} b\right\rangle^{(4)}-\sum_{i j=1}^{N}\left\langle a_{i}^{\dagger} a_{j}\right\rangle^{(4)}\right)-2 \hbar \Delta \mu \sum_{i=1}^{N}\left\langle E_{c}^{\dagger} a_{i}^{\dagger}\right\rangle^{(2)}\left\langle a_{i} a_{i}\right\rangle^{(2)}-i \hbar \sqrt{\frac{\kappa}{2}} \bar{E}_{0}^{c}\left(b_{\mathrm{in}}^{\mathrm{L}}\right)^{\dagger}\langle P\rangle^{(3)}
$$

where we also used that the input fields are classical states uncorrelated with the cavity. Our task is now to express the steady-state cavity photon number $N_{\mathrm{p}}=\left\langle b^{\dagger} b\right\rangle$, total molecular excited-state population $N_{m}=\sum_{i=1}^{N}\left\langle a_{i}^{\dagger} a_{i}\right\rangle$ and intermolecular coherences $\left\langle a_{i}^{\dagger} a_{j}\right\rangle_{i \neq j}$ in terms of the input field operators to the desired orders. The steady-state cavity photon number satisfies

$$
N_{p}^{(4)}=-\frac{2}{\hbar \kappa} \operatorname{Im}\left\langle E_{c}^{\dagger} P\right\rangle^{(4)}-\sqrt{\frac{2}{\kappa}} \operatorname{Re}\left\langle\left(b_{\text {in }}^{L}\right)^{\dagger} b\right\rangle^{(4)},
$$

whereas the total molecular excited-stated population and intermolecular coherences are given by:

$$
\begin{aligned}
& N_{m}^{(4)}=\frac{2}{\hbar \gamma_{m}} \operatorname{Im}\left\langle E_{c}^{\dagger} P\right\rangle^{(4)} \\
& \sum_{i>j} \sum_{j=1}^{N}\left(\left\langle a_{i}^{\dagger} a_{j}\right\rangle^{(4)}+\left\langle a_{j}^{\dagger} a_{i}\right\rangle^{(4)}\right)=\frac{2(N-1)}{\hbar \gamma_{m}} \operatorname{Im}\left\langle E_{c}^{\dagger} P\right\rangle^{(4)}+\frac{4 \Delta}{\gamma_{m}} \sum_{i j=1}^{N} \operatorname{Im}\left[\left\langle a_{j}^{\dagger} a_{j}^{\dagger}\right\rangle^{(2)}\left\langle a_{j} a_{i}\right\rangle^{(2)}\right],
\end{aligned}
$$

where to obtain the last line, we used $\operatorname{Im}\left[\left\langle a_{j}^{\dagger} a_{j}^{\dagger}\right\rangle^{(2)}\left\langle a_{j} a_{j}\right\rangle^{(2)}\right]=\operatorname{Im}\left[\left|\left\langle a_{j}^{\dagger} a_{j}^{\dagger}\right\rangle^{(2)}\right|^{2}\right]=0$. Using the last three results, we find the intermediate result

$$
N N_{p}^{(4)}-\sum_{i j=1}^{n}\left\langle a_{i}^{\dagger} a_{j}\right\rangle^{(4)}=-\frac{2 N}{\hbar \eta} \operatorname{Im}\left\langle E_{c}^{\dagger} P\right\rangle^{(4)}-N \sqrt{\frac{2}{\kappa}} \operatorname{Re}\left\langle\left(b_{\mathrm{in}}^{\mathrm{L}}\right)^{\dagger} b\right\rangle^{(4)}-\frac{4 \Delta}{\gamma_{m}} \sum_{i j=1}^{N} \operatorname{Im}\left[\left\langle a_{j}^{\dagger} a_{j}^{\dagger}\right\rangle^{(2)}\left\langle a_{j} a_{i}\right\rangle^{(2)}\right]
$$

where $\eta^{-1} \equiv \kappa^{-1}+\eta^{-1}$. We now have all of the quantities required to obtain the rate of nonlinear absorption $W$. In particular, it follows from inserting our last result in Eq. 48 that

$$
\begin{aligned}
\left(\hbar \tilde{\omega}_{c}^{*}-\hbar \tilde{\omega}_{0}\right)\left\langle E_{c}^{\dagger} P\right\rangle^{(4)}= & \frac{\Omega_{R}^{2}}{2 \hbar \eta} \operatorname{Im}\left[\left\langle E_{c}^{\dagger} P\right\rangle^{(4)}\right]+\frac{\Omega_{R}^{2}}{4} \sqrt{\frac{2}{\kappa}} \operatorname{Re}\left[\left(b_{\mathrm{in}}^{\mathrm{L}}\right)^{\dagger}\langle b\rangle^{(3)}\right]+\frac{\Omega_{R}^{2} \Delta}{N \gamma_{m}} \sum_{i j=1}^{N} \operatorname{Im}\left[\left\langle a_{j}^{\dagger} a_{j}^{\dagger}\right\rangle\left\langle a_{j} a_{i}\right\rangle\right] \\
& -2 \hbar \Delta \mu \sum_{i=1}^{N}\left\langle a_{i} a_{i}\right\rangle^{(2)}\left\langle a_{i}^{\dagger} E_{c}^{\dagger}\right\rangle^{(2)}-i \hbar \sqrt{\frac{\kappa}{2}} \bar{E}_{0}^{c}\left\langle b_{\mathrm{in}}^{\mathrm{L}}\right\rangle^{\dagger}\langle P\rangle^{(3)}
\end{aligned}
$$

where we used $\Omega_{R}=2\left|\mu E_{0}^{c}\right| \sqrt{N}$. Note the 1.h.s of the previous equation can be written as:

$$
\begin{aligned}
\left(\hbar \tilde{\omega}_{c}^{*}-\hbar \tilde{\omega}_{0}\right)\left[\operatorname{Re}\left\langle E_{c}^{\dagger} P\right\rangle^{(4)}+i \operatorname{Im}\left\langle E_{c}^{\dagger} P\right\rangle^{(4)}\right]= & \left(\hbar \omega_{c}-\hbar \omega_{0}\right) \operatorname{Re}\left[\left\langle E_{c}^{\dagger} P\right\rangle^{(4)}\right]-\frac{\hbar \eta_{s}}{2} \operatorname{Im}\left[\left\langle E_{c}^{\dagger} P\right\rangle^{(4)}\right] \\
& +i\left[\frac{\hbar \eta_{s}}{2} \operatorname{Re}\left(\left\langle E_{c}^{\dagger} P\right\rangle^{(4)}\right)+\left(\hbar \omega_{c}-\hbar \omega_{0}\right) \operatorname{Im}\left(\left\langle E_{c}^{\dagger} P\right\rangle^{(4)}\right)\right] .
\end{aligned}
$$


where we introduced the notation $\eta_{s}=\kappa+\gamma_{m}$. Using the above to equate the imaginary part of the left and right-hand-side of Eq. 53, we find that:

$$
\begin{aligned}
\operatorname{Re}\left(\left\langle E_{c}^{\dagger} P\right\rangle^{(4)}\right)+\frac{\hbar\left(\omega_{c}-\omega_{0}\right)}{\eta_{s}} & W^{(4)}=-\frac{4 \Delta \mu N}{\eta_{s}} \operatorname{Im}\left[\left\langle a_{i} a_{i}\right\rangle^{(2)}\left\langle a_{i}^{\dagger} E_{c}^{\dagger}\right\rangle^{(2)}\right]-\frac{\sqrt{2 \kappa}}{\eta_{s}} \operatorname{Re}\left[\bar{E}_{0}^{c}\left(b_{\mathrm{in}}^{\mathrm{L}}\right)^{\dagger}\langle P\rangle^{(3)}\right], \\
\left(\omega_{c}-\omega_{0}\right) \operatorname{Re}\left(\left\langle E_{c}^{\dagger} P\right\rangle^{(4)}\right)-\frac{\hbar \eta_{s}}{4} W^{\mathrm{NL}}= & \frac{\Omega_{R}^{2}}{4 \hbar \eta} W^{\mathrm{NL}}+\frac{\Omega_{R}^{2}}{4 \hbar}\left\{\sqrt{\frac{2}{\kappa}} \operatorname{Re}\left[\left(b_{\mathrm{in}}^{\mathrm{L}}\right)^{\dagger}\langle b\rangle^{(3)}\right]+\frac{4 \Delta}{N \gamma_{m}} \sum_{i j=1}^{N} \operatorname{Im}\left[\left\langle a_{j}^{\dagger} a_{j}^{\dagger}\right\rangle^{(2)}\left\langle a_{j} a_{i}\right\rangle^{(2)}\right]\right\} \\
& -2 \Delta \mu \sum_{i=1}^{N} \operatorname{Re}\left[\left\langle a_{i} a_{i}\right\rangle^{(2)}\left\langle a_{i}^{\dagger} E_{c}^{\dagger}\right\rangle^{(2)}\right]+\sqrt{\frac{\kappa}{2}} \operatorname{Im}\left[\bar{E}_{0}^{c}\left\langle b_{\mathrm{in}}^{\mathrm{L}}\right\rangle^{\dagger}\langle P\rangle^{(3)}\right],
\end{aligned}
$$

where we used $W^{\mathrm{NL}} \equiv W^{(4)}=\frac{2}{\hbar} \operatorname{Im}\left(\left\langle E_{c}^{\dagger} P\right\rangle^{(4)}\right)$. We can now eliminate $\operatorname{Re}\left(\left\langle E_{c}^{\dagger} P\right\rangle^{(4)}\right)$ and solve for $W^{\mathrm{NL}}$ in terms of the input field variables. This procedure gives

$$
\begin{aligned}
W^{\mathrm{NL}}= & -\frac{2 \eta \hbar \eta_{s}}{2 \eta\left[\left(\hbar \omega_{c}-\hbar \omega_{0}\right)^{2}+\left(\hbar \eta_{s}\right)^{2} / 4\right]+\Omega_{R}^{2} \eta_{s} / 2} \frac{\Omega_{R}^{2}}{4 \hbar}\left\{\sqrt{\frac{2}{\kappa}} \operatorname{Re}\left[\left(b_{\mathrm{in}}^{\mathrm{L}}\right)^{\dagger}\langle b\rangle^{(3)}\right]+\frac{4 \Delta(N-1)}{\gamma_{m}} \operatorname{Im}\left[\left\langle a_{j}^{\dagger} a_{j}^{\dagger}\right\rangle^{(2)}\left\langle a_{j} a_{i}\right\rangle_{j \neq i}^{(2)}(2 \omega)\right]\right\} \\
& +\frac{2 \eta \hbar \eta_{s}}{2 \eta\left[\left(\hbar \omega_{c}-\hbar \omega_{0}\right)^{2}+\left(\hbar \eta_{s}\right)^{2} / 4\right]+\Omega_{R}^{2} \eta_{s} / 2}\left\{2 \Delta \mu N \operatorname{Re}\left[\left\langle a_{i} a_{i}\right\rangle^{(2)}\left\langle a_{i}^{\dagger} E_{c}^{\dagger}\right\rangle^{(2)}\right]+\sqrt{\frac{\kappa}{2}} \operatorname{Im}\left[\bar{E}_{0}^{c}\left\langle b_{\mathrm{in}}^{\mathrm{L}}\right\rangle^{\dagger}\langle P\rangle^{(3)}\right]\right\} \\
& +\frac{2 \eta \hbar \eta_{s}\left(\omega_{c}-\omega_{0}\right)}{2 \eta\left[\left(\hbar \omega_{c}-\hbar \omega_{0}\right)^{2}+\left(\hbar \eta_{s}\right)^{2} / 4\right]+\Omega_{R}^{2} \eta_{s} / 2}\left\{\frac{4 \Delta \mu N}{\eta_{s}} \operatorname{Im}\left[\left\langle a_{i} a_{i}\right\rangle^{(2)}\left\langle a_{i}^{\dagger} E_{c}^{\dagger}\right\rangle\right]+\frac{\sqrt{2 \kappa}}{\eta_{s}} \operatorname{Re}\left[\bar{E}_{0}^{c}\left(b_{\mathrm{in}}^{\mathrm{L}}\right)^{\dagger}\langle P\rangle^{(3)}\right]\right\},(57)
\end{aligned}
$$

where we used that $\operatorname{Im}\left[\left\langle a_{j}^{\dagger} a_{j}^{\dagger}\right\rangle\left\langle a_{j} a_{i}\right\rangle\right]=0$ when $i=j$. Thus, our final expression for the total nonlinear absorption is given by:

$$
\begin{aligned}
W^{\mathrm{NL}}(\omega)= & -\frac{\eta \Omega_{R}^{2} \eta_{s}}{2 \eta\left[\left(\hbar \omega_{c}-\hbar \omega_{0}\right)^{2}+\left(\hbar \eta_{s}\right)^{2} / 4\right]+\Omega_{R}^{2} \eta_{s} / 2} \operatorname{Re}\left[\sqrt{\frac{1}{2 \kappa}}\left[b_{\mathrm{in}}^{\mathrm{L}}(\omega)\right]^{\dagger}\langle b\rangle^{(3)}(\omega)\right] \\
& -\frac{\eta \Omega_{R}^{2} \eta_{s}}{2 \eta\left[\left(\hbar \omega_{c}-\hbar \omega_{0}\right)^{2}+\left(\hbar \eta_{s}\right)^{2} / 4\right]+\Omega_{R}^{2} \eta_{s} / 2} \frac{2 \Delta(N-1)}{\gamma_{m}} \operatorname{Im}\left[\left\langle a_{j}^{\dagger} a_{j}^{\dagger}\right\rangle^{(2)}(-2 \omega)\left\langle a_{j} a_{i}\right\rangle_{j \neq i}^{(2)}(2 \omega)\right] \\
& +\frac{2 \eta(2 \hbar \Delta N) \eta_{s}}{2 \eta\left[\left(\hbar \omega_{c}-\hbar \omega_{0}\right)^{2}+\left(\hbar \eta_{s}\right)^{2} / 4\right]+\Omega_{R}^{2} \eta_{s} / 2} \operatorname{Re}\left[\left\langle a_{i}^{\dagger} a_{i}^{\dagger}\right\rangle^{(2)}(-2 \omega)\left\langle\mu a_{i} E_{c}\right\rangle^{(2)}(2 \omega)\right] \\
& +\frac{\eta \eta_{s}}{2 \eta\left[\left(\hbar \omega_{c}-\hbar \omega_{0}\right)^{2}+\left(\hbar \eta_{s}\right)^{2} / 4\right]+\Omega_{R}^{2} \eta_{s} / 2} \operatorname{Im}\left[\hbar \sqrt{2 \kappa} \bar{E}_{0}^{c}\left[b_{\mathrm{in}}^{\mathrm{L}}(\omega)\right]^{\dagger}\langle P\rangle^{(3)}(\omega)\right] \\
& +\frac{2 \eta\left(\hbar \omega_{c}-\hbar \omega_{0}\right) 4 \Delta N}{2 \eta\left[\left(\hbar \omega_{c}-\hbar \omega_{0}\right)^{2}+\left(\hbar \eta_{s}\right)^{2} / 4\right]+\Omega_{R}^{2} \eta_{s} / 2} \operatorname{Im}\left[\left\langle a_{i} a_{i}\right\rangle^{(2)}(2 \omega)\left\langle\mu a_{i}^{\dagger} E_{c}^{\dagger}\right\rangle^{(2)}(-2 \omega)\right] \\
& +\frac{2 \eta\left(\hbar \omega_{c}-\hbar \omega_{0}\right) \sqrt{2 \kappa}}{2 \eta\left[\left(\hbar \omega_{c}-\hbar \omega_{0}\right)^{2}+\left(\hbar \eta_{s}\right)^{2} / 4\right]+\Omega_{R}^{2} \eta_{s} / 2} \operatorname{Re}\left[\bar{E}_{0}^{c}\left[b_{\mathrm{in}}^{\mathrm{L}}(\omega)\right]^{\dagger}\langle P\rangle^{(3)}(\omega)\right] .
\end{aligned}
$$

Each of the above terms can be further simplified by using results obtained in Sec. 2. For instance, the identities $\langle b\rangle^{(3)}(\omega)=-\bar{E}_{0}^{c} G_{p p}^{(0)}(\omega)\langle P\rangle^{(3)}(\omega)$ and $i \bar{E}_{0}^{c}\left[b_{\mathrm{in}}^{\mathrm{L}}(\omega)\right]^{\dagger}=\sqrt{\frac{\kappa}{2}} \sqrt{\frac{2 \mathcal{F}}{\pi}} E_{\mathrm{in}}^{(-)}(\omega)$ (see Eq. 14) can be employed to simplify the first line of the last equation, while the $2 \mathrm{nd}$, 3rd, and 5 th lines can be simplified using the following results from Eqs. 30 and 33

$$
\begin{aligned}
& \left\langle a_{i} a_{j}\right\rangle_{i \neq j}^{(2)}(\omega)=-\frac{\mu E_{0}^{c}}{\hbar \omega-2 \hbar \tilde{\omega}_{0}}\left[\left\langle a_{j} b\right\rangle^{(2)}(\omega)+\left\langle a_{i} b\right\rangle^{(2)}(\omega)\right], \text { and } \\
& \left\langle a_{i} b\right\rangle^{(2)}(\omega)=-\frac{\hbar \omega-2 \hbar \tilde{\omega}_{0}+2 \hbar \Delta}{\mu E_{0}^{c}}\left\langle a_{i} a_{i}\right\rangle^{(2)}(\omega), \text { which imply that } \\
& \Longrightarrow\left\langle a_{i} a_{j}\right\rangle_{i \neq j}^{(2)}(\omega)=\frac{\left(\hbar \omega-2 \hbar \omega_{0}+2 \hbar \Delta+i \hbar \gamma_{m}\right)\left(\hbar \omega-2 \hbar \omega_{0}-i \hbar \gamma_{m}\right)}{\left(\hbar \omega-2 \hbar \omega_{0}\right)^{2}+\hbar^{2} \gamma_{m}^{2}} \times 2\left\langle a_{i} a_{i}\right\rangle^{(2)}(\omega) .
\end{aligned}
$$




\subsection{Zero detuning}

The physical content of the terms in Eq. 58 becomes clearer in the zero-detuning case where $\omega_{c} \approx \omega_{0}$, in which case the last two lines of Eq. 58 vanish. Taking advantage also that when the strong coupling condition is satisfied $\Omega_{R} \gg \hbar \eta$ and $\Omega_{R} \gg \hbar \eta_{s}$, the nonlinear absorption can be written as a sum of four simple contributions

$$
W^{\mathrm{NL}}(\omega)=\sum_{\alpha=1}^{4} W^{\mathrm{NL}_{\alpha}}(\omega)\left|E_{\text {in }}^{(+)}(\omega)\right|^{4}
$$

where

$$
\begin{aligned}
& W^{\mathrm{NL}_{1}}(\omega) \approx-\frac{2 \eta \kappa}{\hbar}\left[\frac{1}{4\left(\omega-\omega_{0}\right)^{2}+\kappa^{2}}+\frac{1}{\left(\Omega_{R} / \hbar\right)^{2}}\right] \operatorname{Re}\left[\sqrt{\frac{2 \mathcal{F}}{\pi}} \chi^{(3)}(\omega)\right] \\
& W^{\mathrm{NL}_{2}}(\omega) \approx \frac{4 \eta}{\hbar} \frac{\omega-\omega_{0}}{4\left(\omega-\omega_{0}\right)^{2}+\kappa^{2}} \operatorname{Im}\left[\sqrt{\frac{2 \mathcal{F}}{\pi}} \chi^{(3)}(\omega)\right] \\
& W^{\mathrm{NL}_{3}}(\omega) \approx \eta \frac{4 \Delta^{2} N}{\left(\omega-\omega_{0}\right)^{2}+\gamma_{m}^{2} / 4} \frac{\left|\left\langle a_{i} a_{i}\right\rangle^{(2)}(2 \omega)\right|^{2}}{\left|E_{\mathrm{in}}^{(+)}(\omega)\right|^{4}}, \\
& W^{\mathrm{NL}_{4}}(\omega) \approx-\eta \frac{\left(2 \omega-\omega_{20}\right) 2 \Delta N}{\left(\Omega_{R} / 2 \hbar\right)^{2}} \frac{\left|\left\langle a_{i} a_{i}\right\rangle^{(2)}(2 \omega)\right|^{2}}{\left|E_{\mathrm{in}}^{(+)}(\omega)\right|^{4}} .
\end{aligned}
$$

\section{NONLINEAR RESPONSE OF BARE MOLECULAR SYSTEM}

In order to describe the free space nonlinear polarization induced on a bare molecule ensemble driven by external continuous-wave fields, we employ an effective Hamiltonian that is similar to that used to model the molecular system in an optical cavity. The main difference is that each molecule now interacts with several EM modes (with vanishing momentum along the $x, y$ directions) quantized with periodic boundary conditions. The total Hamiltonian in the rotating-wave approximation is:

$$
H=\sum_{\omega} \hbar \omega b_{\omega}^{\dagger} b_{\omega}+\sum_{i=1}^{N}\left(\hbar \omega_{0} a_{i}^{\dagger} a_{i}-\hbar \Delta a_{i}^{\dagger} a_{i}^{\dagger} a_{i} a_{i}\right)-\mu \sum_{i=1}^{N} \sum_{\omega>0}\left(E_{0 \omega} a_{i}^{\dagger} b_{\omega} e^{i \omega z_{i} / c}+\bar{E}_{0 \omega} a_{i} b_{\omega}^{\dagger} e^{-i \omega z_{i} / c}\right),
$$

where $\omega=c k, k=2 \pi m / L, m \in \mathbb{Z}$, and $z_{i}$ is the projection of the position of molecule $i$ on the field direction of propagation, and $E_{0 \omega}=i \sqrt{\frac{\hbar \omega}{2 \epsilon_{0} V}}$. The input field which drives the material polarization is introduced as a boundary condition to the electromagnetic mode operators in the Heisenberg picture, i.e., the input field satisfies the homogeneous part of the EM field equations. We assume the $E_{0 \omega}$ are classical variables, as in the computation performed with the optical cavity in the previous sections.

The equation of motion for the expectation value of the molecular polarization is given by:

$$
\left(i \hbar \partial_{t}-\hbar \tilde{\omega}_{0}\right)\left\langle\mu a_{i}(t)\right\rangle=-2 \hbar \Delta \mu\left\langle a_{i}^{\dagger}(t) a_{i}(t) a_{i}(t)\right\rangle-\mu^{2} \sum_{\omega} E_{0 \omega}\left\langle b_{\omega}(t)\right\rangle e^{i \omega z_{i} / c} .
$$

Assuming the system is always in a pure state, the equation of motion for the third-order component of $\left\langle a_{i}(t)\right\rangle$ is given by:

$$
\left(i \hbar \partial_{t}-\hbar \tilde{\omega}_{0}\right)\left\langle a_{i}(t)\right\rangle^{(3)}=-2 \hbar \Delta\left\langle a_{i}^{\dagger}(t)\right\rangle^{(1)}\left\langle a_{i}(t) a_{i}(t)\right\rangle^{(2)} .
$$

The time evolution of the relevant first and second-order molecular expectation values are given by the solutions of the equations

$$
\begin{aligned}
& \left(i \hbar \partial_{t}-\hbar \tilde{\omega}_{0}\right)\left\langle a_{i}(t)\right\rangle^{(1)}=-\mu \sum_{\omega^{\prime}} E_{\omega^{\prime} \text { in }}(t), \\
& \left(i \hbar \partial_{t}-\hbar \tilde{\omega}_{20}\right)\left\langle a_{i}(t) a_{i}(t)\right\rangle^{(2)}=-2 \mu \sum_{\omega^{\prime}}\left\langle a_{i}(t)\right\rangle^{(1)} E_{\omega^{\prime} \text { in }}(t) e^{-i \omega^{\prime} z_{i} / c},
\end{aligned}
$$


where we made the replacement $E_{\omega \text { in }}(t)=E_{0 \omega}\left\langle b_{\omega}(t)\right\rangle$. Using the long wavelength limit, and thus disregarding the spatial dispersion of the electromagnetic field (as in the computations performed for a molecular system in a cavity), the frequency-domain solutions of the prior equations are:

$$
\begin{aligned}
& \left\langle a_{i}\right\rangle^{(1)}(\omega)=-\mu \sum_{\omega_{u}} \frac{E_{\omega_{u} \text { in }}}{\hbar \omega-\hbar \tilde{\omega}_{0}} \delta_{\omega_{u}, \omega}, \\
& \left\langle a_{i} a_{i}\right\rangle^{(2)}(\omega)=\sum_{\omega_{u}} \sum_{\omega_{v}} \frac{2 \mu^{2} E_{\omega_{u} \text { in }} E_{\omega_{v} \text { in }}}{\left(\hbar \omega_{u}+\hbar \omega_{v}-\hbar \tilde{\omega}_{20}\right)\left(\hbar \omega_{u}-\hbar \tilde{\omega}_{0}\right)} \delta_{\omega, \omega_{u}+\omega_{v}},
\end{aligned}
$$

where $\hbar \tilde{\omega}_{20}=\hbar \omega_{20}-i \hbar \gamma_{m}$, and $\hbar \omega_{20}=2 \hbar \omega_{0}-2 \hbar \Delta$ is the energy difference between the doubly-excited vibrational state and the ground-state. These results can also be written in terms of bare molecule single-particle and two-particle retarded response functions in the frequency domain:

$$
\begin{aligned}
& \left\langle a_{i}\right\rangle^{(1)}(\omega)=-\mu G_{m m}^{(0)}(\omega) E_{\omega \mathrm{in}} \\
& \left\langle a_{i} a_{i}\right\rangle^{(2)}(\omega)=\mu^{2} \sum_{\omega_{u}, \omega_{v}} G_{m m, m m}^{(0)}\left(\omega_{u}+\omega_{v}\right) G_{m m}^{(0)}\left(\omega_{u}\right) E_{\omega_{u} \text { in }} E_{\omega_{v} \text { in }} \delta_{\omega, \omega_{u}+\omega_{v}}
\end{aligned}
$$

where $G_{m m}^{(0)}(\omega)=1 /\left(\hbar \omega-\hbar \tilde{\omega}_{0}\right)$ and $G_{m m, m m}^{(0)}(\omega)=2 /\left(\hbar \omega-\hbar \tilde{\omega}_{20}\right)$ are the Fourier transform of the single-particle and two-particle retarded molecular Green functions, respectively. In the time domain, they measure the probability amplitude that a single and a two-phonon state exist for a time $t$ after their creation. Note that the last equation may also be written in terms of a vibration-vibrational scattering matrix element $\Gamma_{m m, m m}^{(0)}(\omega)=\left(\hbar \omega-2 \hbar \tilde{\omega}_{0}\right) /\left(\hbar \omega-2 \hbar \tilde{\omega}_{20}\right)$ as follows

$$
\left\langle a_{i} a_{i}\right\rangle^{(2)}(\omega)=\mu^{2} \sum_{\omega_{u}, \omega_{v}} \Gamma_{m m, m m}^{(0)}\left(\omega_{u}+\omega_{v}\right) G_{m m}^{(0)}\left(\omega_{u}\right) G_{m m}^{(0)}\left(\omega_{v}\right) E_{\omega_{u} \text { in }} E_{\omega_{v} \text { in }} \delta_{\omega, \omega_{u}+\omega_{v}}
$$

Direct insertion of Eqs. 70 and 71 into the frequency-domain representation of Eq. 67 gives the following solution:

$$
\left\langle a_{i}\right\rangle^{(3)}\left(\omega_{s}\right)=4 \mu^{3} \hbar \Delta \sum_{\omega_{u} \omega_{v} \omega_{w}} \frac{E_{\omega_{u} \text { in }} \bar{E}_{\omega_{w} \text { in }} E_{\omega_{v} \text { in }}}{\left(\hbar \omega_{s}-\hbar \tilde{\omega}_{0}\right)\left(\hbar \omega_{w}-\hbar \tilde{\omega}_{0}^{*}\right)\left(\hbar \omega_{u}+\hbar \omega_{v}-\hbar \tilde{\omega}_{20}\right)\left(\hbar \omega_{u}-\hbar \tilde{\omega}_{0}\right)} \delta_{\omega_{s}, \omega_{u}+\omega_{v}-\omega_{w}} .
$$

In terms of the bare molecule Green functions and phonon-phonon scattering amplitudes, the bare third-order molecular nonlinear polarization $P_{0}^{(3)}\left(\omega_{s}\right)=\mu \sum_{i=1}^{N}\left\langle a_{i}\left(\omega_{s}\right)\right\rangle^{(3)}$ can be written as

$$
\langle P\rangle_{0}^{(3)}\left(\omega_{s}\right)=\sum_{\omega_{u} \omega_{v} \omega_{w}} 2 \hbar \Delta N \mu^{4} G_{m m}^{(0)}\left(\omega_{s}\right) \bar{G}_{m m}^{(0)}\left(\omega_{w}\right) \Gamma_{m m, m m}^{(0)}\left(\omega_{u}+\omega_{v}\right) G_{m m}\left(\omega_{v}\right) G_{m m}\left(\omega_{u}\right) E_{\omega_{u} \text { in }} \bar{E}_{\omega_{w} \text { in }} E_{\omega_{v} \text { in }} \delta_{\omega_{s}, \omega_{u}+\omega_{v}-\omega_{w}},
$$

which implies the bare nonlinear susceptibility

$$
\chi_{0}^{(3)}\left(-\omega_{s} ; \omega_{v},-\omega_{w}, \omega_{u}\right)=2 \hbar \Delta N \mu^{4} G_{m m}^{(0)}\left(\omega_{s}\right) \bar{G}_{m m}^{(0)}\left(\omega_{w}\right) \Gamma_{m m, m m}^{(0)}\left(\omega_{u}+\omega_{v}\right) G_{m m}^{(0)}\left(\omega_{v}\right) G_{m m}^{(0)}\left(\omega_{u}\right) \delta_{\omega_{s}, \omega_{v}-\omega_{w}+\omega_{u}} .
$$

\section{NONLINEAR ABSORPTION SPECTRUM OF BARE MOLECULAR SYSTEM}

The steady-state rate of photon absorption by the molecular system in free space can be computed from the Hamiltonian in Eq. 65. In particular, the steady-state condition stipulates that in the presence of an external radiation field, the rate of excitation of the molecular system is equal to its rate of decay, and therefore $\partial_{t} \sum_{i=1}^{N}\left\langle a_{i}^{\dagger}(t) a_{i}(t)\right\rangle=0$, where $t$ is an arbitrary time during which the system satisfies the condition given above.

Using Heisenberg-Langevin equations of motion for the description of the response of the molecular system to the external electromagnetic field we find that:

$$
\gamma_{m} \sum_{i=1}^{N}\left\langle a_{i}^{\dagger}(t) a_{i}(t)\right\rangle=\sum_{i=1}^{N} \sum_{\omega} \operatorname{Im}\left[\frac{2 \mu \bar{E}_{0 \omega}}{\hbar}\left\langle b_{\omega}^{\dagger}(t) a_{i}(t)\right\rangle\right] .
$$

The 1.h.s of the above equality corresponds to energy extracted from (or transferred to) the molecular system by the bath, whereas the r.h.s describes the pumping of the molecular system by the electromagnetic field. Assuming the 
usual weak coupling condition to be valid in free space, and taking the external field to be given by a macroscopic coherent state with negligible quantum fluctuations, it follows that the bare rate of photon absorption is given by:

$$
W_{0}=\frac{2}{\hbar} \sum_{\omega} \operatorname{Im}\left[\bar{E}_{\omega \mathrm{in}}(t)\langle P(t)\rangle_{0}\right],
$$

where $\langle P(t)\rangle_{0}$ refers to the free space (weakly coupled to the EM field) molecular polarization, i.e., $\langle P(t)\rangle_{0}=$ $\left\langle\sum_{i=1}^{N} \mu a_{i}(t)\right\rangle_{0}$. Thus, the nonlinear contribution to the molecular absorption spectrum is given by:

$$
W_{0}^{\mathrm{NL}}=\frac{2}{\hbar} \operatorname{Im}\left[\bar{E}_{\mathrm{in}}\left(t_{\mathrm{ss}}\right)\left\langle P\left(t_{\mathrm{ss}}\right)\right\rangle_{0}^{(3)}\right]
$$

where $t_{\mathrm{ss}}$ is sufficiently long that the system is in steady-state. Equivalently, we can write

$$
\begin{aligned}
W_{0}^{\mathrm{NL}}(\omega) & =\frac{2}{\hbar} \operatorname{Im} \sum_{\omega_{s}} \bar{E}_{\omega_{s} \text { in }}\left\langle P\left(\omega_{s}\right)\right\rangle_{0}^{(3)} \\
& =\frac{2}{\hbar} \sum_{\omega_{s}} \sum_{\omega_{u} \omega_{v} \omega_{w}} \operatorname{Im}\left[\chi_{0}^{(3)}\left(-\omega_{s} ; \omega_{v},-\omega_{w}, \omega_{u}\right) \bar{E}_{\omega_{s} \text { in }} E_{\omega_{u} \text { in }} \bar{E}_{\omega_{w} \text { in }} E_{\omega_{v} \text { in }}\right] \delta_{\omega_{s}, \omega_{v}-\omega_{w}+\omega_{u}}
\end{aligned}
$$

The nonlinear absorption spectrum for photons with frequency $\omega$ is given by:

$$
W_{0}^{\mathrm{NL}}(\omega)=\frac{2\left|E_{\omega \mathrm{in}}\right|^{4}}{\hbar} \operatorname{Im}\left[\chi_{0}^{(3)}(-\omega ; \omega,-\omega, \omega)\right] .
$$

Using Eq. 76, we find the nonlinear rate of absorption of photons by the molecular system is given by:

$$
\begin{aligned}
W_{0}^{\mathrm{NL}}(\omega) & =N \frac{2}{\hbar} \frac{4 \hbar \Delta \mu^{4}}{\left[\left(\hbar \omega-\hbar \omega_{0}\right)^{2}+\hbar^{2} \gamma_{m}^{2} / 4\right]^{2}} \frac{\hbar \gamma_{m}\left(\hbar \omega_{0}-\hbar \omega\right)}{\left(2 \hbar \omega-\hbar \omega_{20}\right)^{2}+\hbar^{2} \gamma_{m}^{2}}\left|E_{\omega \text { in }}\right|^{4} \\
& +N \frac{2}{\hbar} \frac{4 \hbar \Delta \mu^{4}}{\left[\left(\hbar \omega-\hbar \omega_{0}\right)^{2}+\hbar^{2} \gamma_{m}^{2} / 4\right]^{2}} \frac{\hbar \gamma_{m}\left(\hbar \omega_{20} / 2-\hbar \omega\right)}{\left(2 \hbar \omega-\hbar \omega_{20}\right)^{2}+\hbar^{2} \gamma_{m}^{2}}\left|E_{\omega \text { in }}\right|^{4} .
\end{aligned}
$$

Each of the two terms in the above rate of nonlinear absorption correspond to a distinct nonlinear absorption resonance. This can be seen by noting that the first term vanishes when $\omega=\omega_{0}$, whereas the second vanishes when $2 \omega$ is resonant with the two-photon transition with frequency $\omega_{20}=2 \omega_{0}-2 \Delta$. When $\Delta / \gamma_{m} \gg 1$, the lineshapes corresponding to the two possible nonlinear absorption resonances are well separated, and we can isolate the contribution to $W_{0}^{\mathrm{NL}}(\omega)$ corresponding to two-photon absorption:

$$
W_{0}^{\mathrm{TPA}}(\omega) \equiv N \frac{2}{\hbar} \frac{4 \hbar \Delta \mu^{4}}{\left[\left(\hbar \omega-\hbar \omega_{0}\right)^{2}+\hbar^{2} \gamma_{m}^{2} / 4\right]^{2}} \frac{\hbar \gamma_{m}\left(\hbar \omega_{0}-\hbar \omega\right)}{\left(2 \hbar \omega-\hbar \omega_{20}\right)^{2}+\hbar^{2} \gamma_{m}^{2}}\left|E_{\omega \mathrm{in}}\right|^{4}
$$

The textbook expression for the two-photon absorption rate [6] follows from the last result by taking the limit where $\Delta \gg \gamma$, and by assuming only probe frequencies $\omega$ around the TPA resonance at $\omega_{0}-\Delta$ (so that no other quantum transitions interference with the absorption) In this case, it follows that ${ }^{5}$ :

$$
W_{0}^{\mathrm{TPA}}(\omega) \approx \frac{2 \pi N}{\hbar} \frac{2 \mu^{4}}{\left(\hbar \omega-\hbar \omega_{0}\right)^{2}} \rho_{2}(2 \omega)\left|E_{\omega \mathrm{in}}\right|^{4}
$$

where $\rho_{2}(2 \omega)=-\frac{1}{\pi} \operatorname{Im}\left[G_{m m, m m}^{(2)}(2 \omega)\right]$ :

$$
\rho_{2}(2 \hbar \omega)=-\frac{1}{\pi} \operatorname{Im}\left[\frac{2}{2 \hbar \omega-\hbar \omega_{20}+i \hbar \gamma}\right]=\frac{1}{\pi} \frac{2 \hbar \gamma}{\left(2 \hbar \omega-\hbar \omega_{20}\right)^{2}+\hbar^{2} \gamma_{m}^{2}} .
$$

$$
\begin{aligned}
& { }^{5} \text { Specifically, letting } \omega=\omega_{0}-\Delta-\epsilon \text { with } \epsilon \rightarrow 0 \text { and } \gamma_{m} / \Delta \rightarrow 0 \text {, we have } \\
& \qquad \begin{aligned}
\frac{\hbar \Delta\left(\hbar \omega_{0}-\hbar \omega\right)}{\left[\left(\hbar \omega-\hbar \omega_{0}\right)^{2}+\hbar^{2} \gamma_{m}^{2} / 4\right]^{2}} & \approx \frac{\hbar^{2} \Delta^{2}(1+\epsilon / \Delta)}{\left[\left(\hbar \omega-\hbar \omega_{0}\right)^{2}\right]^{2}} \\
& \approx \frac{\hbar^{2} \Delta^{2}}{\left(\hbar \omega-\hbar \omega_{0}\right)^{2}} \frac{(1+\epsilon / \Delta)}{\hbar^{2} \Delta^{2}(1+\epsilon / \Delta)^{2}} \\
& =\frac{1}{\left(\hbar \omega-\hbar \omega_{0}\right)^{2}}[1+O(\epsilon / \Delta)] .
\end{aligned}
\end{aligned}
$$



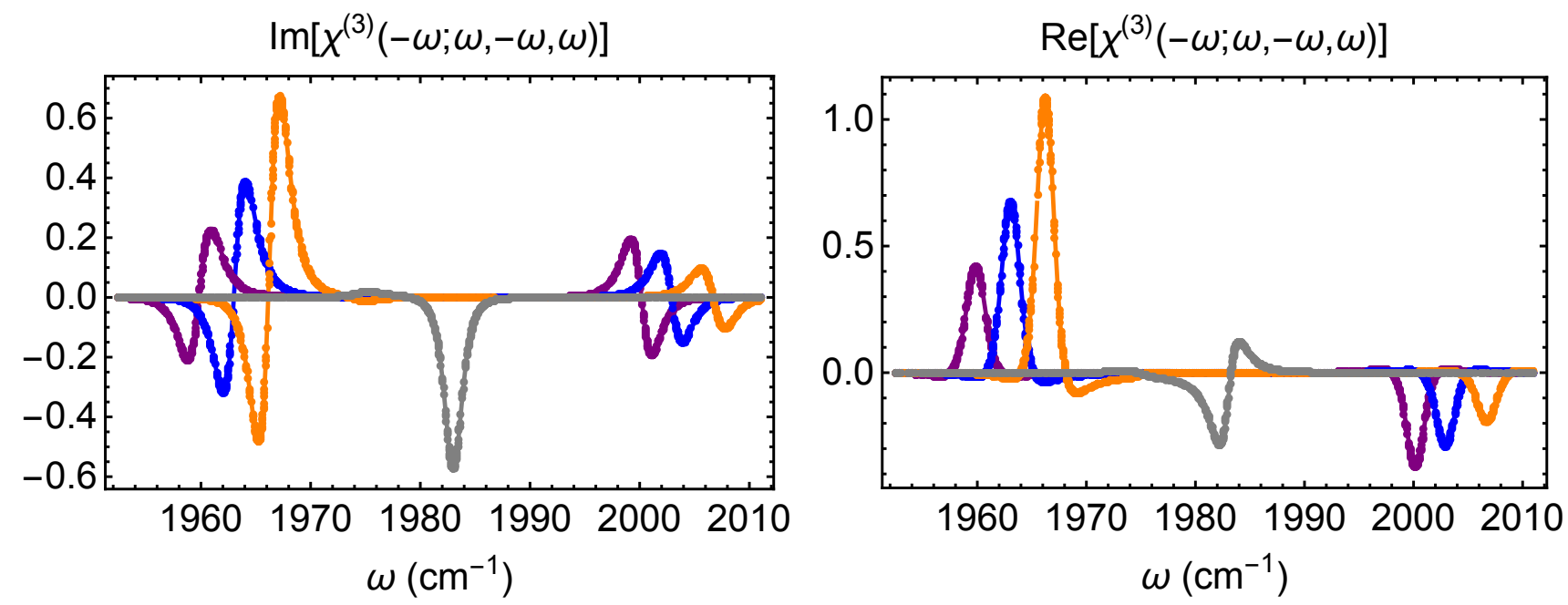

FIG. 1. Left (Right): Imaginary (Real) parts of $\chi^{(3)}(-\omega ; \omega,-\omega, \omega)$ and $\chi_{0}^{(3)}(-\omega ; \omega,-\omega, \omega)$ for a system with $\omega_{0}=$ $1983 \mathrm{~cm}^{-1}, \gamma_{m}=3 \mathrm{~cm}^{-1}, \kappa=6 \mathrm{~cm}^{-1}, \Omega_{R}=40 \mathrm{~cm}^{-1}$, and $\Delta=8 \mathrm{~cm}^{-1}$. The grey curve corresponds to results obtained for the bare molecular system. Purple (orange) corresponds to $\omega_{c}-\omega_{0}=7 \mathrm{~cm}^{-1}\left(\omega_{c}-\omega_{0}=-7 \mathrm{~cm}^{-1}\right)$, and blue describes the results obtained for $\omega_{c}=\omega_{0}$.

\section{QUANTITATIVE COMPARISON OF MOLECULAR NONLINEAR SUSCEPTIBILITY OF BARE AND STRONGLY COUPLED SYSTEMS}

In this section, we provide a quantitative discussion of the molecular nonlinear susceptibility under strong coupling with a cavity. In Fig. 1, we employ a prototypical system of $\mathrm{W}(\mathrm{CO})_{6}$ molecules in hexane [7] with $\omega_{0}=1983 \mathrm{~cm}^{-1}$, $\gamma_{m}=3 \mathrm{~cm}^{-1}, \Delta=8 \mathrm{~cm}^{-1}$ to illustrate and compare the real and imaginary parts of the bare nonlinear susceptibility (Eq. 76) to that obtained for the same system under strong coupling with an optical cavity (Eq. 44) with $\kappa=6 \mathrm{~cm}^{-1}$, $\Omega_{R}=40 \mathrm{~cm}^{-1}$, and the following cavity frequencies: $\omega_{c}=1977 \mathrm{~cm}^{-1}, 1983 \mathrm{~cm}^{-1}, 1990 \mathrm{~cm}^{-1}$ [8-10]. For the sake of simplicity, we show results for a monochromatic input field with frequency $\omega$ (thus, $\omega_{u}=\omega_{w}=\omega_{w}=\omega$ ).

Fig. 1 shows that the bare and strongly coupled molecular system display strikingly contrasting nonlinear polarization. The imaginary part of the bare nonlinear susceptibility shows absorptive lineshapes, whereas dispersive behavior can be observed for the polaritonic. The opposite is true for the corresponding real parts. The absorptive lineshapes for $\operatorname{Im}\left[\chi_{0}^{(3)}(-\omega ; \omega,-\omega, \omega)\right]$ centered at $\omega_{0}$ and $\omega_{0}-\Delta$ (see small bump of grey curve around $\omega=1975 \mathrm{~cm}^{-1}$ ) are expected since this function is directly proportional to the nonlinear absorption rate (Eq. 81) by the bare molecules. The weak resonance at $\omega_{0}-\Delta$ corresponds to two-photon absorption by the molecular subsystem which absorb two input photons with $\omega=\omega_{0}-\Delta$ to generate a population of molecules with energy $\hbar \omega=2 \hbar \omega_{0}-2 \hbar \Delta$ in the doubly-excited state, whereas the resonance at $\omega_{0}$ results from stimulated emission by excited-state population and ground-state bleach which contribute to the reduced nonlinear photon absorption probability at the fundamental frequency $\omega_{0}$ (thus giving rise to the observed negative amplitude).

It is harder to interpret $\operatorname{Im}\left[\chi^{(3)}(-\omega ; \omega,-\omega, \omega)\right]$. As discussed in Sec. 3, by virtue of the cavity-matter strong coupling, the nonlinear polarization contribution to the energy absorbed by the molecular subsystem is not directly proportional to the imaginary part of $\chi^{(3)}(-\omega ; \omega,-\omega, \omega)$. Nevertheless, the most obvious features of the molecular nonlinear susceptibility under strong coupling are visible from Fig. 1. For instance, the absorptive lineshapes displayed by $\operatorname{Re}\left[\chi^{(3)}(-\omega ; \omega,-\omega, \omega)\right]$ are all centered at the LP and the UP frequencies for each of the studied systems. Stronger nonlinear polarization always happens at $\omega=\omega_{\mathrm{LP}}$ in comparison to $\omega=\omega_{\mathrm{UP}}$. This happens because, while for $N \gg 1$, the nonlinear response mediated by LP and UP arises mainly from their interaction with molecular doubly excitedstates (see Sec. 7), larger spectral overlap exists between the molecular two-photon transition and the $\mathrm{LP}_{2}$ resonance (for the parameters here chosen). As a result, energy or amplitude transfer between polaritons and molecular doubly excited-states is more efficient when the LP is resonantly driven by the external field (see detailed discussion and connection to experiments [9] in Sections 3 and 4 of the main text).

Note also that, for the parameters chosen to obtain Fig. 1, the maxima of the nonlinear susceptibility obtained for the molecular system inside and outside of an optical cavity are of the same order of magnitude. However, we expect that if $\Omega_{R}$ is modified so that two-polariton states $\left(\mathrm{LP}_{2}\right.$ in this example) become nearly-resonant with molecular doubly excited-states, the molecular nonlinear susceptibility under strong coupling will likely undergo significant 

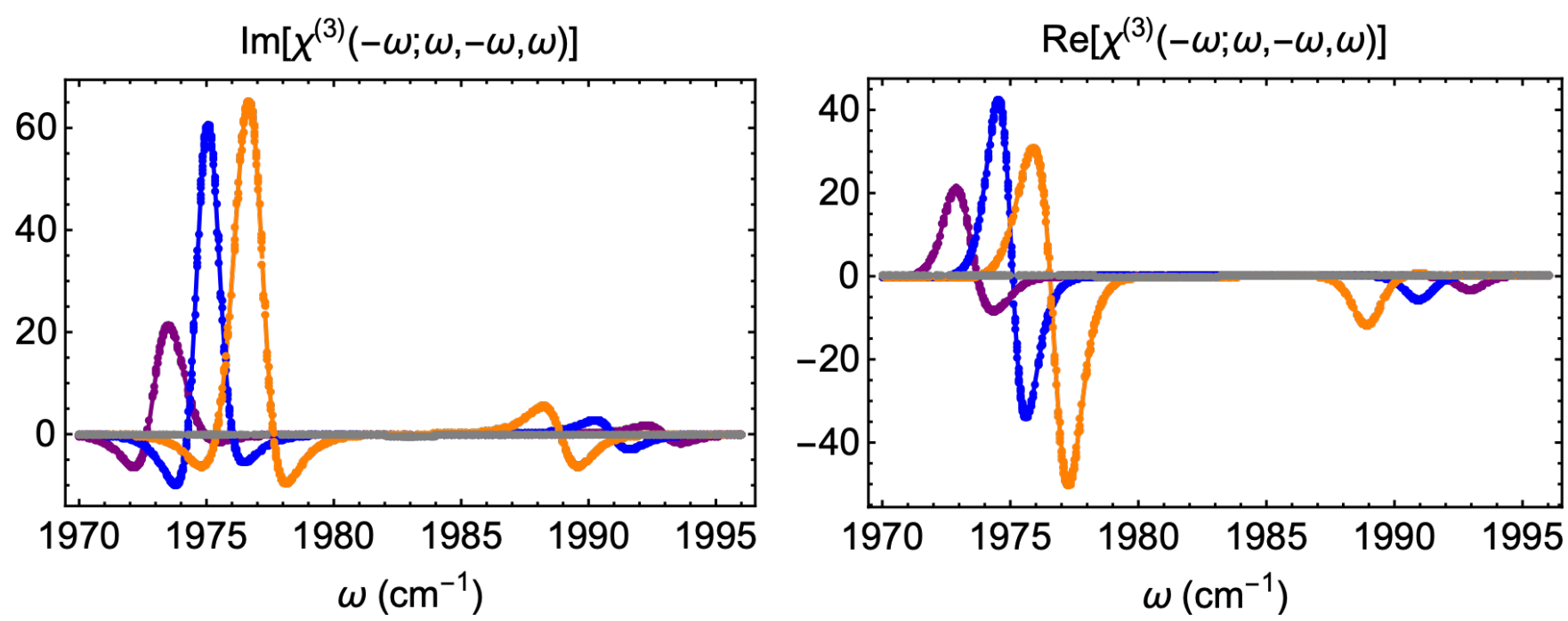

FIG. 2. Left (Right): Imaginary (Real) parts of $\chi^{(3)}(-\omega ; \omega,-\omega, \omega)$ and $\chi_{0}(-\omega ; \omega,-\omega, \omega)$ for a system with equal cavity and molecular fundamental frequencies and decay rates and varying Rabi splitting. The barely visible grey curve corresponds to results obtained for the bare molecular system, whereas the purple, blue, and orange correspond to $\Omega_{R}=20,16$, and $12 \mathrm{~cm}^{-1}$.

enhancement, since in this case, spectral overlap between $\mathrm{LP}_{2}$ and molecular doubly excited-states will be large, and the latter will provide an efficient sink for energy disposal by the former (this is not the case for any of the scenarios shown in Fig. 1).

We conclude this section by presenting in Fig. 2 the behavior of the strongly coupled molecular nonlinear susceptibility for $\Omega_{R}=20,16$, and $12 \mathrm{~cm}^{-1}$ for a system with zero real and imaginary detuning $\left(\omega_{c}=\omega_{0}=1983 \mathrm{~cm}^{-1}\right.$ and $\kappa=\gamma=3 \mathrm{~cm}^{-1}$, respectively) and $\Delta=8 \mathrm{~cm}^{-1}$. Our expectation of an enhanced molecular nonlinear susceptibility under strong coupling with a moderate quality cavity is now verified. Figure 2 shows that as $\Omega_{R}-2 \Delta \rightarrow 0$, the nonlinear polarization of the molecular subsystem becomes larger, especially when the two-LP frequency $2 \omega_{0}-\Omega_{R}$ approaches the TPA resonance at $2 \omega=2 \omega_{0}-2 \Delta$. We can observe enhancement of both real and imaginary parts of $\chi^{(3)}$ relative to $\chi_{0}^{(3)}$ by two orders of magnitude at $\omega=\omega_{0}-2 \Delta$ when the condition $\Omega_{R}=2 \Delta$ is satisfied. Note that $\operatorname{Im}\left[\chi^{(3)}(-\omega ; \omega,-\omega, \omega)\right]$ has absorptive lineshapes at the TPA transition. This feature suggests that the enhanced signal at $\omega=\omega_{\text {LP }}$ is due to two-LP decay into molecular doubly excited-states. This channel is discussed in detail in Secs. 3 and 4 of the main manuscript.

\section{ENERGY EIGENVALUES AND EIGENSTATES OF NON-DISSIPATIVE SYSTEM}

In this section, we obtain the optical spectrum of the hybrid system discussed in the main manuscript. For this purpose, we neglect the effects of dissipation, so that the obtained transition frequencies are real. In fact, the Hamiltonian of the hybrid system can be written in this case as:

$$
H=\hbar \omega_{c} b^{\dagger} b+\sum_{i=1}^{N} \hbar \omega_{0} a_{i}^{\dagger} a_{i}-\hbar \Delta \sum_{i=1}^{N} a_{i}^{\dagger} a_{i}^{\dagger} a_{i} a_{i}-\sum_{i=1}^{N} \hbar g\left(a_{i}^{\dagger} b+b^{\dagger} a_{i}\right),
$$

where $g$ is the single-molecule light-matter coupling constant. two conservation laws follow from the effective Hamiltonian given in the main text. First, the Hamiltonian is invariant under permutation of the molecules. Thus, the eigenstates of $H$ can be classified according to the irreducible representation fo the permutation group of $N$ symbols $\left(S_{N}\right)$, and time-dependent evolution only allows transitions between states which belong to the same irrep. Second, it follows from the RWA approximation to the light-matter interaction that the Hamiltonian evolution of the composite system preserves the total number of excitations of the photonic and matter subsystems $M=\sum_{i=1}^{N} a_{i}^{\dagger} a_{i}+b^{\dagger} b$. Therefore, the eigenstates of $H$ may also be classified according to the total number of excitations in the molecular and photonic subsystems. For instance, the ground-state of the system $(M=0)$ has all molecules in the ground-state, while the cavity field is in its vacuum state. The states with $M=1$ contain either a single excited vibration $\left(\left|1_{i}\right\rangle\right.$ where $1 \leq i \leq N)$, or a single-photon $\left(\left|1_{0}\right\rangle\right)$, etc. 
Of the many irreps of $S_{N}$, only the totally-symmetric is relevant in our case. In the manifold of states with $M=1$, this feature is well-known: only the totally-symmetric superposition of states with a single excited molecule exchanges energy with the cavity field. The non-totally-symmetric states are dark and thus provide no contribution to the optical response of the hybrid system (in the studied ideal model).

The lower and upper polariton states are denoted by $|\mathrm{LP}\rangle$ and $|\mathrm{UP}\rangle$. They can be written in terms of the local-mode basis states as follows:

$$
\begin{aligned}
& |\mathrm{LP}\rangle=-\sin (\theta / 2)\left|1_{0}\right\rangle+\cos (\theta / 2)\left|1_{S}\right\rangle, \\
& |\mathrm{UP}\rangle=\cos (\theta / 2)\left|1_{0}\right\rangle+\sin (\theta / 2)\left|1_{S}\right\rangle,
\end{aligned}
$$

where $2 \theta=\tan ^{-1}\left[2 g \sqrt{N} /\left(\omega_{c}-\omega_{0}\right)\right]$ and $\left|1_{S}\right\rangle=N^{-1 / 2} \sum_{i=1}^{N}\left|1_{i}\right\rangle$ is the molecular singly-excited bright state, and we denote by $g$ the single-molecule light-matter coupling.

The bright subspace of the doubly-excited state $(M=2)$ manifold contains the four two-particle (hybrid) states which are totally-symmetric under permutation of the molecular labels. These states are the only which can be accessed via two-photon transitions in our model (dark modes are never accessed since they require molecular permutational symmetry-breaking operators which are disregarded in our treatment). They are given by:

$$
\left|2_{0}\right\rangle, \quad\left|1_{0} 1_{m}\right\rangle=\frac{1}{\sqrt{N}} \sum_{a=1}^{N}\left|1_{0} 1_{a}\right\rangle, \quad\left|1_{m} 1_{m^{\prime}}\right\rangle_{m \neq m^{\prime}}=\sqrt{\frac{2}{N(N-1)}} \sum_{a>b}\left|1_{a} 1_{b}\right\rangle, \quad\left|2_{m}\right\rangle=\frac{1}{\sqrt{N}} \sum_{a=1}^{N}\left|2_{a}\right\rangle .
$$

Figure S1 illustrates how Hamiltonian evolution induces transitions between these states. From this figure, we can also see that these four states are the only which can be accessed from a two-photon initial state. In the subspace spanned by the priorly defined states, the total Hamiltonian is given by:

$$
H_{2}^{\mathrm{B}}(N)=\left(\begin{array}{cccc}
2 \hbar \omega_{c} & \hbar g \sqrt{2 N} & 0 & 0 \\
\hbar g \sqrt{2 N} & \hbar \omega_{0}+\hbar \omega_{c} & \hbar g \sqrt{2(N-1)} & \hbar g \sqrt{2} \\
0 & \hbar g \sqrt{2(N-1)} & 2 \hbar \omega_{0} & 0 \\
0 & \hbar g \sqrt{2} & 0 & 2 \hbar \omega_{0}-2 \hbar \Delta
\end{array}\right),
$$

where the matrix was ordered in the same way as the basis states in Eq. 90. From now on, we will focus on the case where $\omega_{c} \approx \omega_{0}$ since this gives the simplest analytical results, and is also the most relevant.

If the molecular oscillators were two-level systems, we would obtain the restriction of the Tavis-Cummings Hamiltonian to the $M=2$ Hamiltonian, which is given by:

$$
H_{2 \mathrm{TC}}^{\mathrm{B}}(N)=\left(\begin{array}{ccc}
2 \hbar \omega_{c} & \hbar g \sqrt{2 N} & 0 \\
\hbar g \sqrt{2 N} & \hbar \omega_{0}+\hbar \omega_{c} & \hbar g \sqrt{2(N-1)} \\
0 & \hbar g \sqrt{2(N-1)} & 2 \hbar \omega_{0}
\end{array}\right) .
$$

When $\omega_{0}=\omega_{c}$, the TC eigenstates can be readily obtained since the secular equation can be written in the simple form:

$$
\left(2 \omega_{0}-\lambda\right)\left[\left(2 \omega_{0}-\lambda\right)^{2}-2(N-1) g^{2}\right]-2 g^{2} N\left(2 \omega_{0}-\lambda\right)=0,
$$

which has solutions:

$$
\begin{aligned}
& \lambda_{\mathrm{UP}}^{\mathrm{TC}}=2 \omega_{0}+2 g \sqrt{N-1 / 2} \approx 2 \omega_{\mathrm{UP}}-\frac{g}{2 \sqrt{N}}, \\
& \lambda_{\mathrm{LP}}^{\mathrm{TC}}=2 \omega_{0}-2 g \sqrt{N-1 / 2} \approx 2 \omega_{\mathrm{LP}}+\frac{g}{2 \sqrt{N}}, \\
& \lambda_{\mathrm{LU}}^{\mathrm{TC}}=2 \omega_{0},
\end{aligned}
$$

where the approximate expressions resulted from taking the limit where $N \rightarrow \infty$. In terms of the bare states $\left|2_{0}\right\rangle$, $\left|1_{0} 1_{m}\right\rangle$ and $\left|1_{m} 1_{m^{\prime}}\right\rangle$, the eigenstates corresponding to the above energies are given by:

$$
\begin{aligned}
\left|\mathrm{UP}_{2}^{\mathrm{TC}}\right\rangle & =\sqrt{\frac{N}{4 N-2}}\left|2_{0}\right\rangle+\sqrt{\frac{1}{2}}\left|1_{0} 1_{m}\right\rangle+\sqrt{\frac{N-1}{4 N-2}}\left|1_{m} 1_{m^{\prime}}\right\rangle \\
\left|\mathrm{LP}_{2}^{\mathrm{TC}}\right\rangle & =\sqrt{\frac{N}{4 N-2}}\left|2_{0}\right\rangle-\sqrt{\frac{1}{2}}\left|1_{0} 1_{m}\right\rangle+\sqrt{\frac{N-1}{4 N-2}}\left|1_{m} 1_{m^{\prime}}\right\rangle \\
\left|\mathrm{LU}^{\mathrm{TC}}\right\rangle & =\sqrt{\frac{N-1}{2 N-1}}\left|2_{0}\right\rangle-\sqrt{\frac{N}{2 N-1}}\left|1_{m} 1_{m^{\prime}}\right\rangle .
\end{aligned}
$$




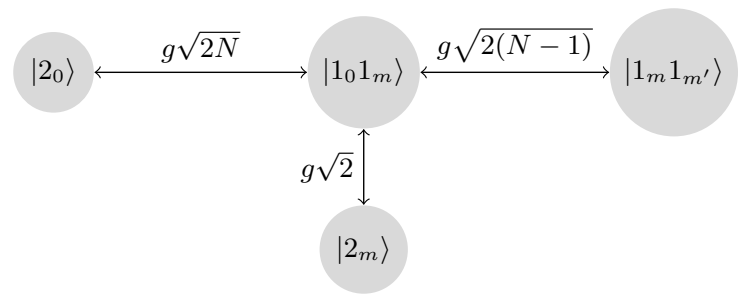

FIG. 3. Scheme representing the bright (totally-symmetric matter and cavity states) two-particles states which play a role in the nonlinear spectroscopy of vibrational polaritons discussed here. Above each arrow connecting a pair of states we provide the corresponding Hamiltonian matrix elements (coupling constants).

Using these states along with the $\left|2_{i}\right\rangle$ as the new basis vectors for the totally-symmetric doubly-excited manifold of the system allowing two excitations, the Hamiltonian matrix (with the row and column indices in the order $\left.\left|\mathrm{LU}^{\mathrm{TC}}\right\rangle,\left|\mathrm{UP}_{2}^{\mathrm{TC}}\right\rangle,\left|\mathrm{LP}_{2}^{\mathrm{TC}}\right\rangle,\left|2_{i}\right\rangle\right)$, acquires the simple form:

$$
H_{2}^{\mathrm{B}}(N)=\left(\begin{array}{cccc}
2 \hbar \omega_{0} & 0 & 0 & 0 \\
0 & \hbar \lambda_{\mathrm{UP}}^{\mathrm{TC}} & 0 & \hbar g \\
0 & 0 & \hbar \lambda_{\mathrm{LP}_{2}}^{\mathrm{TC}} & -\hbar g \\
0 & \hbar g & -\hbar g & 2 \hbar \omega_{0}-2 \hbar \Delta
\end{array}\right), \quad \hbar \omega_{c}=\hbar \omega_{0} .
$$

From this, we can see that the state $\left|\mathrm{LU}^{\mathrm{TC}}\right\rangle$ is also an eigenstate of the complete Hamiltonian, and that despite its delocalization, the totally-symmetric doubly-excited molecular state $\left|2_{S}\right\rangle$ is only weakly-coupled to polaritons (the corresponding coupling constant is given by the single-molecule light-matter interaction energy $g$ ). If we take the singlemolecule light-matter coupling to be very weak compared to the energy differences $\lambda_{\mathrm{UP}_{2}}^{\mathrm{TC}}-\left(2 \omega_{0}-2 \Delta\right)=g \sqrt{4 N-2}+2 \Delta$ and $\lambda_{\mathrm{LP}_{2}}^{\mathrm{TC}}-\left(2 \omega_{0}-2 \Delta\right)=-g \sqrt{4 N-2}+2 \Delta$, we can obtain reasonable approximate eigenstates and eigenvalues of $H_{2}^{\mathrm{B}}(N)$. This will almost always be a valid assumption, even if $2 \Delta$ is nearly equal to $g \sqrt{4 N-2}$, since the singlemolecule-light coupling constant $g$ is generally too small compared to the energy scale of vibrational motion, and there exists a large number of (non-totally symmetric) molecular doubly excited-states with energy $2 \omega_{0}-2 \Delta$ that provides an efficient decay channel for $\mathrm{LP}_{2}$ states. In other words, the TC eigenstates will almost always be very good approximations to the eigenstates of $H_{2}^{\mathrm{B}}(N)$. The leading-order perturbatively-corrected eigenvalues are given by:

$$
\begin{aligned}
& \omega_{\mathrm{UP}_{2}} \approx 2 \omega_{0}+g \sqrt{4 N-2}+\frac{1}{2} \frac{2 g^{2}}{g \sqrt{4 N-2}+2 \Delta} \approx 2 \omega_{\mathrm{UP}}-\frac{g}{2 \sqrt{N}}+\frac{1}{2} \frac{2 g^{2}}{g \sqrt{4 N-2}+2 \Delta}, \\
& \omega_{\mathrm{LP}_{2}} \approx 2 \omega_{0}-g \sqrt{4 N-2}-\frac{1}{2} \frac{2 g^{2}}{g \sqrt{4 N-2}-2 \Delta} \approx 2 \omega_{\mathrm{LP}}+\frac{g}{2 \sqrt{N}}-\frac{1}{2} \frac{2 g^{2}}{g \sqrt{4 N-2}-2 \Delta}, \\
& \omega_{2_{S}} \approx 2 \omega_{0}-2 \Delta+\Delta \frac{g^{2}}{g^{2}(N-1 / 2)-\Delta^{2}}, \\
& \omega_{\mathrm{LU}}=2 \omega_{0},
\end{aligned}
$$

where we included the exact eigenvalue of the $|\mathrm{LU}\rangle$ state for completeness. The corresponding approximate eigenstates can be written as:

$$
\begin{aligned}
& \left|\mathrm{UP}_{2}\right\rangle \approx \sqrt{\frac{N}{4 N-2}}\left|2_{0}\right\rangle+\sqrt{\frac{1}{2}}\left|1_{0} 1_{m}\right\rangle+\sqrt{\frac{N-1}{4 N-2}}\left|1_{m} 1_{m^{\prime}}\right\rangle+\frac{g}{g \sqrt{4 N-2}+2 \Delta}\left|2_{m}\right\rangle, \\
& \left|\mathrm{LP}_{2}\right\rangle \approx \sqrt{\frac{N}{4 N-2}}\left|2_{0}\right\rangle-\sqrt{\frac{1}{2}}\left|1_{0} 1_{m}\right\rangle+\sqrt{\frac{N-1}{4 N-2}}\left|1_{m} 1_{m^{\prime}}\right\rangle+\frac{g}{g \sqrt{4 N-2}-2 \Delta}\left|2_{m}\right\rangle, \\
& \left|2_{S}\right\rangle \approx\left|2_{m}\right\rangle-\frac{g^{2}}{g^{2}(2 N-1)-2 \Delta^{2}}\left(\sqrt{N}\left|2_{0}\right\rangle+\sqrt{N-1}\left|1_{m} 1_{m^{\prime}}\right\rangle\right)+\frac{\sqrt{2} g \Delta}{g^{2}(2 N-1)-2 \Delta^{2}}\left|1_{0} 1_{m}\right\rangle, \\
& |\mathrm{LU}\rangle=\sqrt{\frac{N-1}{2 N-1}}\left|2_{0}\right\rangle-\sqrt{\frac{N}{2 N-1}}\left|1_{m} 1_{m^{\prime}}\right\rangle .
\end{aligned}
$$


[1] C. W. Gardiner and M. J. Collett, "Input and output in damped quantum systems: Quantum stochastic differential equations and the master equation," Physical Review A 31, 3761-3774 (1985).

[2] Daniel A Steck, Quantum and Atom Optics, revision 0.12 .0 ed. (available online at http://atomopticsnas.uoregon.edu/ ${ }^{\sim}$ dsteck/teaching/quantum-optics/quantum-optics-notes.pdf, 2017).

[3] Daniel A Steck, Classical and Modern Optics, revision 1.7.4 ed. (available online at http://steck.us/teaching, 2017).

[4] Alexey V Kavokin, Jeremy J Baumberg, Guillaume Malpuech, and Fabrice P Laussy, Microcavities, Vol. 21 (Oxford University Press, 2017).

[5] Shaul Mukamel, Principles of Nonlinear Optical Spectroscopy (Oxford University Press on Demand, 1999).

[6] R.W. Boyd and D. Prato, Nonlinear Optics (Elsevier Science, 2008).

[7] Bo Xiang, Raphael F. Ribeiro, Adam D. Dunkelberger, Jiaxi Wang, Yingmin Li, Blake S. Simpkins, Jeffrey C. Owrutsky, Joel Yuen-Zhou, and Wei Xiong, "Two-dimensional infrared spectroscopy of vibrational polaritons," Proceedings of the National Academy of Sciences, 201722063 (2018).

[8] Raphael F. Ribeiro, Adam D. Dunkelberger, Bo Xiang, Wei Xiong, Blake S. Simpkins, Jeffrey C. Owrutsky, and Joel Yuen-Zhou, "Theory for Nonlinear Spectroscopy of Vibrational Polaritons," The Journal of Physical Chemistry Letters 9, 3766-3771 (2018).

[9] Bo Xiang, Raphael F. Ribeiro, Liying Chen, Jiaxi Wang, Matthew Du, Joel Yuen-Zhou, and Wei Xiong, "State-Selective Polariton to Dark State Relaxation Dynamics," The Journal of Physical Chemistry A 123, 5918-5927 (2019).

[10] Bo Xiang, Raphael F. Ribeiro, Yingmin Li, Adam D. Dunkelberger, Blake B. Simpkins, Joel Yuen-Zhou, and Wei Xiong, "Manipulating optical nonlinearities of molecular polaritons by delocalization," Science Advances 5, eaax5196 (2019). 